\title{
GRASSMANN GEOMETRIES IN INFINITE DIMENSIONAL HOMOGENEOUS SPACES AND AN APPLICATION TO REFLECTIVE SUBMANIFOLDS
}

\author{
DAVID BRANDER
}

\begin{abstract}
Let $U$ be a real form of a complex semisimple Lie group, and $(\tau, \sigma)$ a pair of commuting involutions on $U$. This data corresponds to a reflective submanifold of a symmetric space, $U / K$. We define an associated integrable system, and describe how to produce solutions from curved flats. This gives many new examples of submanifolds as integrable systems.

The solutions are shown to correspond to various special submanifolds, depending on which homogeneous space, $U / L$, one projects to. We apply the construction to a question which generalizes, to the context of reflective submanifolds of arbitrary symmetric spaces, the problem of isometric immersions of space forms with negative extrinsic curvature and flat normal bundle. For this problem, we prove that the only cases where local solutions exist are the previously known cases of space forms, in addition to our new example of constant curvature Lagrangian immersions into complex projective and complex hyperbolic spaces. We also prove non-existence of global solutions in the compact case.

For other reflective submanifolds, lower dimensional solutions exist, and can be described in terms of Grassmann geometries. We consider one example in detail, associated to the group $G_{2}$, obtaining a special class of surfaces in $S^{6}$.
\end{abstract}

\section{INTRODUCTION}

The primary aim of this paper is to understand an integrable system characterized by a loop group associated to any pair of commuting involutions on a real semisimple Lie group. This generalizes a system which was introduced by Ferus and Pedit [13] to study isometric immersions of space forms. From this generalization, new examples of special submanifolds as integrable systems can be obtained. The application which we will focus on is a natural analogue, pertaining to an arbitrary reflective submanifold in a symmetric space, of the problem of isometric immersions of space forms with negative extrinsic curvature and flat normal bundle.

2000 Mathematics Subject Classification. Primary 37K10, 37K25, 53C42, 53B25; Secondary 53C35.

Key words and phrases. Integrable systems; Loop groups; Symmetric spaces; Reflective submanifolds. 
1.1. Background. A reflective submanifold $N$, of a connected Riemannian manifold $P$, is a totally geodesic symmetric submanifold. Reflective submanifolds of Riemannian symmetric spaces, $P=U / K$, were classified by DSP Leung [19, 20, 21]. Each can be characterized by a pairwise symmetric Lie algebra, $(\mathfrak{u}, \tau, \sigma)$, where $\tau$ and $\sigma$ are commuting involutions, and $\tau$ determines the symmetric space $U / K$. Let $\mathfrak{u}=\mathfrak{k} \oplus \mathfrak{p}=\mathfrak{u}_{+} \oplus \mathfrak{u}_{-}$be the canonical decompositions of the Lie algebra, $\mathfrak{u}$, induced by $\tau$ and $\sigma$ respectively. Then $N$ is the totally geodesic submanifold through the origin, $K$, obtained by exponentiating $\mathfrak{p}^{\prime}:=\mathfrak{p} \cap \mathfrak{u}_{-}$. Clearly there is a second (semi-Riemannian) symmetric space involved here, $U / U_{+}$, associated to $\sigma$.

As part of the project of classifying symmetric submanifolds of symmetric spaces (see [23, 4] for this story), H Naitoh was led to the use of Grassmann geometries, a concept defined by Harvey and Lawson [14].

If $\mathfrak{G}$ is an arbitrary subset of the Grassmann bundle of tangential $s$-planes over $P$, that is, $G r_{s}(T P)=\cup_{x \in P} G r_{s}\left(T_{x} P\right)$, then a $\mathfrak{G}$-submanifold, $N$, of $P$ is an $s$-dimensional connected submanifold such that $T_{x} N$ is an element of $\mathfrak{G}$ for each $x \in N$. The set of such submanifolds is called the $\mathfrak{G}$-geometry. For us, $P$ will be a homogeneous space, $G / H$, and $\mathfrak{G}$ an orbit of the action of the identity component, $I_{0}(G)$, of $G$ on $G r_{s}(T P)$.

A $\mathfrak{G}$-geometry is called strongly curvature invariant if, for each $s$-plane $W \in \mathfrak{G}$, both $W$ and its orthogonal complement $W^{\perp}$ are invariant under the curvature tensor of $P$. For each strongly curvature invariant $\mathfrak{G}$-geometry of a symmetric space, $U / K$, there is precisely one reflective submanifold, up to isometries of $U / K$, and vice versa. We will be interested in certain nontotally geodesic $\mathfrak{G}$-submanifolds. For this, a result of Naitoh, 24, 25, 27, 26], is helpful (see [4]): the following list contains all strongly curvature invariant $\mathfrak{G}$-geometries which admit non-totally geodesic $\mathfrak{G}$-submanifolds, for the case that $P$ is a simply connected, irreducible symmetric space of compact type:

(i) $k$-dimensional submanifolds of the sphere $S^{n}, 0<k<n$;

(ii) $k$-dimensional complex submanifolds of the complex projective space $\mathbb{C} P^{n}, 0<k<n$

(iii) $n$-dimensional totally real submanifolds of $\mathbb{C} P^{n}, n \geq 2$;

(iv) $2 n$-real-dimensional totally complex submanifolds of the quaternionic projective space $\mathbb{H} P^{n}, n \geq 2$;

(v) The geometries associated with irreducible symmetric $R$-spaces;

There is a precisely analogous list for the non-compact case.

1.2. Problem Statement. If $E_{1}$ and $E_{2}$ are vector bundles over a manifold $M$, with connections $\Gamma_{1}$ and $\Gamma_{2}$ respectively, then we will call the bundle connection pairs $\left(E_{1}, \Gamma_{1}\right)$ and $\left(E_{2}, \Gamma_{2}\right)$ isomorphic if there exists a vector bundle isomorphism $\phi: E_{1} \rightarrow E_{2}$ such that $\phi^{*} \Gamma_{2}=\Gamma_{1}$. Suppose $M$ is an immersed Riemannian submanifold of $N_{1}$, and $f: M \rightarrow N_{2}$ is an isometric immersion, where $\operatorname{Dim}\left(N_{1}\right)=\operatorname{Dim}\left(N_{2}\right)$. We will say that $f$ is normal curvature preserving if the associated normal bundle connection pairs are isomorphic. 
For a Riemannian manifold $M$, let $M_{R}$ denote the same manifold with the metric scaled by a factor of $R>0$. If $P:=U / K$, is a symmetric space, and $N:=\exp \left(\mathfrak{p}^{\prime}\right)$ is a totally geodesic submanifold through the origin, $0:=K$, whose tangent space at 0 is $\mathfrak{p}^{\prime}$, denote the associated orbit on $G r_{\operatorname{Dim}\left(\mathfrak{p}^{\prime}\right)}(T P)$ by $\mathfrak{G}_{\mathfrak{p}^{\prime}}$.

Problem 1.1. Suppose given a simply connected, immersed, reflective submanifold $N:=\exp \left(\mathfrak{p}^{\prime}\right)$ of a semisimple Riemannian symmetric space $P$. Thus, $N_{R}$ is a reflective submanifold of $P_{R}$. Does there exist a (local or global) isometric immersion of $N_{R}$ into $P$ as a normal curvature preserving $\mathfrak{G}_{\mathfrak{p}^{\prime}}$-submanifold? More specifically, we consider this problem for

(i) $R>1$, if $P$ is of compact type;

(ii) $R<1$, if $P$ is of non-compact type.

In the case that $P$ has constant curvature, these ranges for $R$ are equivalent to the requirement that $N_{R}$ has negative extrinsic curvature. On the other hand, for positive extrinsic curvature, one has umbilic hypersurfaces $N_{R}$, so there is no issue there.

Problem 1.1 generalizes the questions of whether there is a local isometric embedding with flat normal bundle of a sphere $S_{R}^{k}$, of radius $\sqrt{R}$ into $S^{n}$, for $R>1$, and the analogue for a hyperbolic space $H_{R}^{k}$ into $H^{n}$ for $R<1$. It is known for these special cases that solutions exist if and only if $n \geq 2 k-1$, and, moreover, that the integrability condition is an integrable system of nonlinear PDE, solutions of which can be obtained by solving a collection of commuting linear differential equations [1, 31, 32, 13. For the sphere, even though local solutions exist, there is no complete global solution [22]. For the hyperbolic case, $H_{R}^{k} \rightarrow H^{n}$, for $R<1$, global non-immersibility is also conjectured to be the case (usually stated in codimension $k-1$ ). It is equivalent to the complete isometric immersion problem with flat normal bundle of hyperbolic space into Euclidean space. Non-immersibility for $H^{2}$ into $E^{3}$ was proven by Hilbert [15]. However, in the simply connected case, the problem remains open for higher dimensions.

1.3. Approach. We consider a generalization of a Grassmann submanifold: given a subset, $\mathfrak{G}$, of $G r_{s}(T P)$, a sub-G-submanifold, or $\mathfrak{G}$-compatible submanifold is an $r$-dimensional submanifold of $P, r \leq s$, each of whose tangent spaces is contained in an element of $\mathfrak{G}$. The basic idea is, given two homogeneous spaces, $G / H$ and $G / K$, where $K$ is a subgroup of $H$, we construct sub-Ğ-submanifolds in $G / K$ which project to $\mathfrak{G}^{\prime}$-submanifolds of $G / H$, for certain geometries $\mathfrak{G}$ and $\mathfrak{G}^{\prime}$. In Section 1.6 below, we explain that, in this article, we are doing precisely that, in an infinite dimensional homogeneous space.

We study $\mathfrak{G}_{-1}^{1}$-compatible submanifolds, where $\mathfrak{G}_{-1}^{1}$ is a particular Grassmann geometry of finite dimensional submanifolds of an infinite dimensional homogeneous space $\mathcal{H} / \mathcal{H}^{0}$. Here $\mathcal{H}$ is a subgroup of the loop group $\Lambda G$ of maps from the unit circle into a complexification, $G$, of $U$. The subgroup 
$\mathcal{H}$ is defined as the fixed point set of three involutions of $\Lambda G$, associated to the pairwise symmetric Lie algebra $(\mathfrak{u}, \tau, \sigma)$. This is a modification and generalization of the set-up used in $[13$.

In Section 2 we outline how to produce $\mathfrak{G}$-compatible submanifolds for a more general class of Grassmann geometries, which contains ours, using a generalized Krichever-Dorfmeister-Pedit-Wu (KDPW) method. This reduces the problem to one of producing simpler objects, which are then susceptible to the standard Adler-Kostant-Symes (AKS) theory, [2, 3, 17, 30, 9]. These methods are well-known to experts in the field.

In Section 3 we describe the three-involution loop group and the $\mathfrak{G}_{-1^{-}}^{1}$ compatible submanifolds, and work out the basic equations satisfied by them. In general, a $\mathfrak{G}_{-1}^{1}$-compatible immersion takes values in the subgroup consisting of loops which have holomorphic extensions to the punctured plane $\mathbb{C}^{*}$. In our case, for a fixed value of the loop parameter $\lambda \in \mathbb{R}^{*}$, the solutions are special (immersed) submanifolds of the homogeneous space $\frac{U}{K \cap U_{+}}$, satisfying certain conditions.

1.4. Results. In Section 4 we study the projections of these immersions to $U / K$, and then to $U / U_{+}$. For the first case, we show, Theorem 4.1, that, if the dimension of the submanifold is equal to the dimension of $\mathfrak{p}^{\prime}$, then regular solutions are precisely the solutions to Problem 1.1. for the case $R>1$ (irrespective of whether the symmetric space $P$ is compact).

For the projections to $U / U_{+}$, we show, Proposition 4.1, that these are curved flats, that is maps which are, at each point, tangent to a flat of $U / U_{+}$. We can then deduce that there is no complete immersion (other than a curve) in the compact case:

Corollary 1.2. Let $N$ and $P$ be as in Problem 1.1, for the case $P$ compact and $R>1, \Sigma_{R} \subset N_{R}$ an open submanifold, and $f: \Sigma_{R} \rightarrow P$ an isometric normal curvature preserving $\mathfrak{G}_{\mathfrak{p}^{\prime}}$-immersion. If the symmetric space $N$ is not flat, then $\Sigma_{R}$ cannot be the whole of $N_{R}$.

In Section 5 we study the regularity of the solutions obtained via the KDPW method, with the following result, which is basic to all that follows:

Theorem 1.3. Let $N:=\exp \left(\mathfrak{p}^{\prime}\right) \subset P=U / K$ be a reflective submanifold, where the associated second symmetric space $U / U_{+}$is Riemannian, and let $R>1$. Then there exists a local isometric immersion, $N_{R} \rightarrow P$, as a normal curvature preserving $\mathfrak{G}_{\mathfrak{p}^{\prime}}$-submanifold, if and only if $\operatorname{dim}(N) \leq$ $\operatorname{Rank}\left(U / U_{+}\right)$.

Note that the assumptions that $R>1$ and $U / U_{+}$is Riemannian are essential for the "only if" direction.

1.4.1. The Compact Case. In Section 6 we check the rank condition for all relevant cases and conclude:

Theorem 1.4. The following list contains the geometric interpretations of all possible local solutions to Problem 1.1 for the case $R>1$ and $P$ is a 
simply connected, compact, irreducible, Riemannian symmetric space. In all cases, local solutions exist and can be constructed by the AKS-KDPW scheme. In all cases where $\operatorname{Dim}\left(N_{R}\right)>1$, there is no solution which is geodesically complete.

(i) $N_{R}=S_{R}^{k}$ is an isometric immersion with flat normal bundle from a $k$-sphere of radius $\sqrt{R}$ into the unit sphere $S^{n}$, with $0<k \leq$ $(n+1) / 2$, and $n \geq 2$.

(ii) $N_{R}=S_{R}^{n}$ is an isometric totally real immersion from an $n$-sphere of radius $\sqrt{R}$ into complex projective space $\mathbb{C} P^{n}$, with $n \geq 2$.

1.4.2. The Non-Compact Case. In Section 7 we consider Problem 1.1 for the non-compact case. Here the second symmetric space, $U / U_{+}$is nonRiemannian, which makes it less straightforward to obtain non-existence results. However, we can use the property of the three involution loop group, explored in [6, 5, that a solution for one problem for a value of the loop parameter $\lambda$ in $\mathbb{R}^{*}$ corresponds to a solution to a different problem for $\lambda$ in $S^{1}$ or $i \mathbb{R}^{*}$. This allows us, in those cases where it is necessary, to equate the problem with one in which $U / U_{+}$is Riemannian. Since the submanifolds are hyperbolic, we do not, however, get global non-immersibility, as we did in the compact case.

Theorem 1.5. The following list contains the geometric interpretations of all possible local solutions to Problem 1.1 for the case $R<1$ and $P$ is a simply connected, irreducible, Riemannian symmetric space of non-compact type. In all cases, local solutions exist and can be constructed by the AKS$K D P W$ scheme.

(i) $N_{R}=H_{R}^{k}$ is an isometric immersion with flat normal bundle from a $k$-dimensional hyperbolic space of constant curvature $\frac{-1}{R}$ into the standard hyperbolic space $H^{n}$, with $0<k \leq(n+1) / 2$, and $n \geq 2$.

(ii) $N_{R}=H_{R}^{n}$ is an isometric totally real immersion from the hyperbolic space of constant curvature $\frac{-1}{R}$ into complex hyperbolic space $\mathbb{C} H^{n}$, with $n \geq 2$.

Remark 1.6. It seems an interesting question to ask whether or not there exist complete immersions in the second case of Theorem 1.5, given the above-mentioned conjectures concerning the first case.

1.5. Other Projections. In the last section we discuss the case where $\operatorname{Dim}\left(\mathfrak{p}^{\prime}\right)>\operatorname{Rank}\left(U / U_{+}\right)$. In this case, the loop group still provides special submanifolds, but they are of dimension at most equal to the rank of $U / U_{+}$, so the projections to $U / K$ are deformations of certain submanifolds of symmetric submanifolds, in fact $s u b-\mathfrak{G}_{\mathfrak{p}^{\prime}}$-submanifolds. There are many of these, as the list of reflective submanifolds contains over one hundred examples [19]. We consider one example only, associated to a pair of involutions on $G_{2}$, and project the solutions, which are of dimension 2 , to the sphere $S^{6}$. The resulting surfaces are deformations of totally geodesic 
complex curves in $S^{6}$, with the property that the restriction of $T S^{6}$ to the surface decomposes canonically into three 2-dimensional sub-bundles, each of which is invariant under the almost complex structure of $S^{6}$.

1.6. Concluding Remarks. The $\mathfrak{G}_{-1}^{1}$-compatible submanifolds here are defined as maps into $\mathcal{H} / \mathcal{H}^{0}$, where $\mathcal{H}^{0}=K \cap U_{+}$. As such, they are only sub$\mathfrak{G}_{-1}^{1}$-submanifolds, and the $\mathfrak{G}_{-1}^{1}$-geometry on this space consists of integral submanifolds for a distribution. However, the final object we are interested in is the associated family of immersions into $U / K$. This can be thought of as a true $\mathfrak{G}_{-1}^{1}$-submanifold in $\mathcal{H} / K$, where this geometry is defined analogously to that on $\mathcal{H} / \mathcal{H}^{0}$, and it is easy to verify that, on $\mathcal{H} / K$, the $\mathfrak{G}_{-1}^{1}$-geometry is not a distribution.

The $\mathfrak{G}_{-1}^{1}$-compatible submanifolds associated to the three involution loop group studied here can, in the real analytic case, be thought of as families of certain pluriharmonic maps [5]. Moreover, the choices of $R$ complementary to those stated in Problem 1.1 can also be obtained from the set-up here. The results for those cases are different, however, as the symmetric space $U / U_{+}$is non-Riemannian.

There are further methods for producing and studying the solutions to the problems described here, such as dressing, and also studying an associated linear problem on an algebraic curve. A possible alternative to the KDPW approach used here would have been the modified AKS construction given in [13.

For global problems, the loop group approach is clearly of use in obtaining non-existence results. On the other hand, the problem of constructing, or proving existence of, solutions with particular global properties is challenging, and more progress in this direction would be interesting.

The basic principal behind this project was to study a naturally occurring integrable system and to interpret the solutions geometrically. This approach has been pursued recently by Terng and collaborators [7, 33].

\section{Integrable Systems Constructed from Loop Groups}

In this section we describe a general type of integrable system, and a way to obtain solutions, using well-established methods. We present it in terms of Grassmann geometries, as this fits well with our applications. The essential fact we will need is the correspondence (4).

2.1. Loop Group Grassmann Geometries. Let $G$ be a complex Lie group, with Lie algebra $\mathfrak{g}$. Let $\Lambda G$ be the group of maps from the unit circle $S^{1}$ into $G$, of a class which includes all loops which extend analytically to some neighbourhood of $S^{1}$, and that makes $\Lambda G$ a Banach Lie group; such as the Wiener topology. $\Lambda G$ includes maps which have holomorphic extensions to $\mathbb{C}^{*}$, and most of the maps we discuss are of this type.

Let $\mathcal{H}$ be a closed subgroup of $\Lambda G$, and $\mathcal{H}^{0}$ be the group of constant loops in $\mathcal{H}$, namely $\mathcal{H} \cap G$. Then the set of left cosets of $\mathcal{H}^{0}$, denoted by $\mathcal{H} / \mathcal{H}^{0}$, is a 
(generally infinite dimensional) homogeneous space on which $\mathcal{H}$ acts by left multiplication. This induces an action on the Grassmann bundle $G r_{s}\left(T \frac{\mathcal{H}}{\mathcal{H}^{0}}\right)$, and so we can talk about associated $\mathfrak{G}$-geometries, where $\mathfrak{G}$ is any orbit of this action.

The Lie algebra $\Lambda \mathfrak{g}$ of $\Lambda G$ consists of the set of Fourier series, $\sum_{i=-\infty}^{\infty} a_{i} \lambda^{i}$, $a_{i} \in \mathfrak{g}$, where $\lambda$ is the $S^{1}$ parameter, and the convergence condition depends on the topology one chooses. The Lie algebra, $\operatorname{Lie}(\mathcal{H})$, of $\mathcal{H}$, is a subalgebra of $\Lambda \mathfrak{g}$, and the Lie algebra, $\operatorname{Lie}\left(\mathcal{H}^{0}\right)$, of $\mathcal{H}^{0}$ is just the set of constant polynomials in $\operatorname{Lie}(\mathcal{H})$. Hence the tangent space at the origin, $0:=\mathcal{H}^{0}$, of $\mathcal{H} / \mathcal{H}^{0}$ consists of the elements of $\operatorname{Lie}(\mathcal{H})$ whose constant terms are zero,

$$
T_{0} \frac{\mathcal{H}}{\mathcal{H}^{0}}=\left\{\sum_{i \neq 0} a_{i} \lambda^{i}\right\} \subset \operatorname{Lie}(\mathcal{H}) .
$$

Given such a subgroup $\mathcal{H}$, define, for integers $a<b, W_{a}^{b}$ to be the vector subspace of $T_{0} \frac{\mathcal{H}}{\mathcal{H}^{0}}$ given by

$$
W_{a}^{b}=\left\{x \in T_{0} \frac{\mathcal{H}}{\mathcal{H}^{0}} \mid x=\sum_{i=a}^{b} a_{i} \lambda^{i}\right\} .
$$

Now set $\mathfrak{G}_{a}^{b}$ to be the distribution given by the orbit of $W_{a}^{b}$ under the action of $\mathcal{H}$ on $G r_{d(b-a)}\left(T \frac{\mathcal{H}}{\mathcal{H}^{0}}\right)$, where $d=\operatorname{Dim}(\mathfrak{g})$.

The basic object we can construct, using the techniques described here, are $\mathfrak{G}_{a}^{b}$-compatible (immersed) submanifolds of $\mathcal{H} / \mathcal{H}^{0}$. They were studied, essentially from this point of view, in [6], where they were called connection order $(a, b)$ maps. This terminology was grounded in the structure of the Maurer-Cartan form of a frame for such an immersion, described below.

Here is a more detailed description: let $M$ be a smooth manifold with a fixed base point $x_{0}$, and denote by $\operatorname{Map}\left(M, x_{0}, \Lambda G, I\right)$ the group of smooth maps $M \rightarrow \Lambda G$, which map $x_{0}$ to the identity. For a smooth map $f$ from $M$ into a Lie group $\mathcal{G}$, we denote by $f^{-1} \mathrm{~d} f$, the pull-back via $f$ of the MaurerCartan form of $\mathcal{G}$. If $F \in \operatorname{Map}\left(M, x_{0}, \Lambda G, I\right)$, then the Maurer-Cartan form $F^{-1} \mathrm{~d} F$ takes its values in the Lie algebra $\Lambda \mathfrak{g}$, that is, it has an expansion

$$
\alpha=F^{-1} \mathrm{~d} F=\sum_{i} \alpha_{i} \lambda^{i}
$$

where $\alpha_{i}$ are $\mathfrak{g}$-valued 1 -forms. For each value of $\lambda$ where $F$ is defined, in particular for all $\lambda \in S^{1}$, we have a smooth map $F_{\lambda}: M \rightarrow G$, with MaurerCartan form $\alpha^{\lambda}$, which must satisfy the integrability condition, known as the Maurer-Cartan equation,

$$
\mathrm{d} \alpha^{\lambda}+\alpha^{\lambda} \wedge \alpha^{\lambda}=0
$$

Conversely, such a family of 1-forms which satisfies (1) on a simply-connected manifold $M$ can be integrated to obtain a unique map $F \in \operatorname{Map}\left(M, x_{0}, \Lambda G, I\right)$. 
If $\mathcal{H}$ is any subgroup of the loop group $\Lambda G$ then, for any extended integers $a, b \in \mathbb{Z} \cup\{ \pm \infty\}$, with $a \leq b$, we define

$\mathcal{M C}(M, \mathcal{H})_{a}^{b}=\left\{F \in \operatorname{Map}\left(M, x_{0}, \mathcal{H}, I\right) \mid F^{-1} \mathrm{~d} F=\sum_{i=a}^{b} \alpha_{i} \lambda^{i}, \alpha_{i}\right.$ constant in $\left.\lambda\right\}$.

The notation " $\mathcal{M C}$ " is meant to remind us that it is the expansion of the Maurer-Cartan form of $F$, not $F$ itself, which is a polynomial of degree $(a, b)$.

Since postmultiplication by an element which is constant in $\lambda$ has no effect on the connection order, we can analogously define

$$
\mathcal{M C}\left(M, \mathcal{H} / \mathcal{H}^{0}\right)_{a}^{b}
$$

If $a$ and $b$ are finite, and an element $f \in \mathcal{M C}\left(M, \mathcal{H} / \mathcal{H}^{0}\right)_{a}^{b}$ is a regular map, $M \rightarrow \mathcal{H} / \mathcal{H}^{0}$, then $f$ is just a $\mathfrak{G}_{a}^{b}$-compatible immersion into $\mathcal{H} / \mathcal{H}^{0}$, mapping $x_{0}$ to the origin. On the other hand, for a fixed value of $\lambda \in \mathbb{C}^{*}$, these are maps, $f^{\lambda}$, from $M$ into some quotient group $\frac{U}{\mathcal{H}^{0}}$, where $U$ is some subgroup of $G$. This leads to the interpretations of $\mathfrak{G}_{a}^{b}$-compatible immersions as families (as $\lambda$ varies) of special submanifolds of a homogeneous space. The fact that the Maurer-Cartan equation (11) holds for all $\lambda$, imposes some conditions on $f_{\lambda}$, which define the special submanifold.

Notation: We sometimes use the same symbol $x$ for an element of $\mathcal{H} / \mathcal{H}^{0}$ as for a representative in $\mathcal{H}$.

2.2. The KDPW Method. This is a general method for constructing a $\mathfrak{G}_{a}^{b}$-compatible immersion, where $a<0<b$, from a pair of simpler objects, namely a $\mathfrak{G}_{a}^{0}$-compatible immersion and a $\mathfrak{G}_{0}^{b}$-compatible immersion, using a Birkhoff factorization of the loop group (see [28]). This is a factorization for $x \in \Lambda G$,

$$
x=x^{+} x^{-},
$$

where the loops $x^{+}$and $x^{-}$have holomorphic extensions to the unit disc $\mathbb{D}_{+}$ and the complement of its closure $\mathbb{D}_{-}$respectively. Denote the respective subgroups of such elements by $\Lambda^{ \pm} G$, and, for a subgroup $\mathcal{H}$, set $\mathcal{H}^{ \pm}:=\mathcal{H} \cap$ $\Lambda^{ \pm} G$. The Birkhoff factorization is valid on an open dense neighbourhood of the identity of $\Lambda G$, and is unique with the normalization $x^{+}(0)=I$. Obviously the analogue holds with \pm interchanged.

The idea was used by Krichever in [18] for solutions of the sine-Gordon equation. A different variant was used for harmonic maps into symmetric spaces by Dorfmeister, Pedit and Wu in [11. In the case of harmonic maps, it reduced the problem to specifying certain holomorphic data, and has since been used successfully to produce numerous examples of constant mean curvature surfaces. This is known as the DPW method. Dorfmeister and the author investigated the general applicability of the basic idea in [6].

If $x$ is an element of a subgroup $\mathcal{H}$ of $\Lambda G$, and the factors $x^{ \pm}$in (2) are also in $\mathcal{H}$, then we say $\mathcal{H}$ is Birkhoff decomposable. In this case, the generalized 
KDPW method described in [6] gives the following bijection:

$$
\begin{aligned}
F \in \mathcal{M C}\left(\Omega, \mathcal{H} / \mathcal{H}^{0}\right)_{a}^{b}, \leftrightarrow \quad & F_{+} \in \mathcal{M C}(\Omega, \mathcal{H})_{1}^{b}, \\
& F_{-} \in \mathcal{M C}(\Omega, \mathcal{H})_{a}^{-1},
\end{aligned}
$$

which holds at least in a neighbourhood, $\Omega$, of the base point, $x_{0}$, of $M$. The maps $F_{+}$and $F_{-}$are simply the unique normalized left factors in the left and right Birkhoff decompositions $F=F_{+} G_{-}=F_{-} G_{+}$.

The version which we will use, is the case that $\mathcal{H}$ is Birkhoff decomposable and we consider a further subgroup, $\mathcal{H}_{\tau}$, where $\tau$ is an involution of the second kind of $\mathcal{H}$, meaning it takes $\mathcal{H}^{ \pm} \rightarrow \mathcal{H}^{\mp}$, and $F \in \mathcal{M C}\left(M, \frac{\mathcal{H}_{\tau}}{\mathcal{H}_{\tau}^{0}}\right)_{a}^{b}$ : then it follows that $a=-b$ and, applying $\tau$ to both sides of the correspondence (3), we see that we must have $F_{-}=\tau F_{+}$. One can show that there is always a bijective correspondence

$$
F \in \mathcal{M C}\left(\Omega, \frac{\mathcal{H}_{\tau}}{\mathcal{H}_{\tau}^{0}}\right)_{-b}^{b} \leftrightarrow F_{+} \in \mathcal{M C}(\Omega, \mathcal{H})_{1}^{b},
$$

for some neighbourhood $\Omega$ of $x_{0}$. If $\mathcal{H}_{\tau}$ is a subgroup of $\Lambda U$, where $U$ is a compact real form of $G$, then we also have a global statement in the right to left direction, because $F$ on the left hand side of (44) is constructed pointwise from an Iwasawa factorization.

2.3. The AKS Theory and Finite Type Solutions. One useful consequence of the correspondence (3) is that the standard AKS theory can be used to generate many solutions which are computed by solving a collection of ODE's on a finite dimensional vector space, so-called finite type solutions. In general, this method applies to $\mathfrak{G}_{a}^{-1}$-compatible and $\mathfrak{G}_{1}^{b}$-compatible immersions, and one can then apply the correspondence (3) to these. A short description can be found in [9]. We will not use this construction here, but wish to point out that this is one means to generate many solutions for the problems studied below.

\section{The Three Involution Loop Group}

3.1. Definition of the Group. Let $G$ be a complex semisimple Lie group, $\tau, \sigma$ a pair of commuting involutions of $G$, and $\rho$ a conjugation of $G$ which commutes with $\tau$ and $\sigma$. Let $U:=G_{\rho}$ be the real form determined by $\rho$, with corresponding Lie algebra $\mathfrak{u}$. Extend the involutions to $\Lambda G$ by the rules:

$$
\begin{array}{r}
(\rho X)(\lambda)=\rho(X(\bar{\lambda})), \\
(\sigma X)(\lambda)=\sigma(X(-\lambda)), \\
(\tau X)(\lambda)=\tau(X(-1 / \lambda)),
\end{array}
$$

and consider the subgroup fixed by all three involutions:

$$
\mathcal{H}=\Lambda G_{\rho \tau \sigma} .
$$

It is easy to show that if $\Lambda G$ is any Birkhoff decomposable group, for example if $G$ is complex semisimple, then so is the subgroup defined as the fixed 
point set of any finite number of commuting finite order automorphisms of the first kind, which include $\rho$ and $\sigma$ defined here. Since $\tau$ is of the second kind, we are in the realm of the second version of the KDPW method and, in particular, can use the correspondence (44).

Now consider a $\mathfrak{G}_{-1}^{1}$-compatible immersion in $\mathcal{H} / \mathcal{H}^{0}$, that is a regular map

$$
F \in \mathcal{M C}\left(M, \mathcal{H} / \mathcal{H}^{0}\right)_{-1}^{1}
$$

For $\lambda \in \mathbb{R}^{*}, F$ is a map from $M$ into $U / U_{\tau \sigma}$, since $\Lambda G_{\rho \tau \sigma}^{0}=U_{\tau \sigma}$. We can also project to obtain maps into the symmetric spaces $U / U_{\tau}$ and $U / U_{\sigma}$, or more generally, into any homogeneous space $U / H$, where $U_{\tau} \cap U_{\sigma} \subset H$.

In principle, we can construct $F$ from elements

$$
F_{+} \in \Lambda G_{\rho \sigma}(M)_{1}^{1}
$$

by the correspondence (44). The maps $F_{+}$are families of curved flats into $U / U_{\sigma}$.

3.2. Curved Flats. Here we summarize the definitions and relevant results from [12, where Ferus and Pedit defined and studied curved flats.

Definition 3.1. If $M$ is a manifold and $N=U / K$ is a (semi-Riemannian) symmetric space, then $f: M \rightarrow N$ is called a curved flat if the pull-back via $f$ of the curvature form on $U / K$ is zero. A curved flat is regular if it is immersive everywhere.

If $\mathfrak{u}=\mathfrak{k} \oplus \mathfrak{p}$ is the canonical decomposition, and the tangent bundle to $N$ is identified with $\mathfrak{p}$ via the differential of left translation from the origin, then the tangent space at each point of a curved flat is an Abelian subspace of $\mathfrak{p}$. Hence if the symmetric space is Riemannian, the maximum possible dimension for a regular curved flat is $\operatorname{Rank}(U / K)$.

If $M$ is simply connected, then curved flats can be characterized as elements of

$$
\mathcal{M C}\left(M, \widehat{\mathcal{H}} / \widehat{\mathcal{H}}^{0}\right)_{0}^{1}, \quad \widehat{\mathcal{H}}:=\Lambda G_{\rho \sigma}
$$

where $\rho$ is the conjugation determining the real form $U$ of a complexification, $G$, of $U$, and $\rho$ is extended to $\Lambda G$ by $(\rho X)(\lambda)=\rho(X(\bar{\lambda}), \sigma$ is extended by the formula $(\sigma X)(\lambda)=\sigma(X(-\lambda))$, and $K$ is the fixed point set of $\left.\sigma\right|_{U}$. To see this, let $F: M \rightarrow U$ be a frame for a curved flat $f$. Note that a global frame may not exist, but one can show that the map into the homogeneous space $\widehat{\mathcal{H}} / \widehat{\mathcal{H}}^{0}$ we are about to construct is nevertheless defined. The Maurer-Cartan form of $F$ has the $\mathfrak{k} \oplus \mathfrak{p}$ decomposition

$$
\alpha=F^{-1} \mathrm{~d} F=\alpha_{\mathfrak{k}}+\alpha_{\mathfrak{p}} .
$$

The curved flat condition is given by

$$
\alpha_{\mathfrak{p}} \wedge \alpha_{\mathfrak{p}}=0 .
$$

It is simple to check that this condition, together with the integrability condition for $\alpha$, namely the Maurer-Cartan equation (1), are equivalent to 
the assumption that the family of 1 -forms,

$$
\alpha^{\lambda}:=\alpha_{\mathfrak{k}}+\lambda \alpha_{\mathfrak{p}},
$$

satisfies the Maurer-Cartan equation for all values of $\lambda$. For $\lambda \in \mathbb{R}^{*}$, we can integrate $\alpha^{\lambda}$ on $M$ to obtain a frame for a curved flat. The associated family is an element of $\mathcal{M C}\left(M, \widehat{\mathcal{H}} / \widehat{\mathcal{H}}^{0}\right)_{0}^{1}$. Note that, if $M$ is contractible, so that a global frame exists, we can gauge away the $\alpha_{\mathfrak{k}}$ term, to get a unique (up to the normalization point $x_{0}$ ) element of $\mathcal{M C}(M, \widehat{\mathcal{H}})_{1}^{1}$.

The results which are of most interest to us are:

\section{Theorem 3.2. 12}

(i) If $\operatorname{Rank}(U / K)=r$, then the AKS scheme can be used to locally construct infinitely many $r$-dimensional curved flats into $U / K$, which are, at each point, tangent to a Cartan subalgebra of $\mathfrak{p}$.

(ii) If $U / K$ is Riemannian, then a curved flat in $U / K$ is intrinsically flat.

The first statement implies that, on $V$, the $\mathfrak{G}_{-1}^{1}$-compatible maps, $F \in$ $\mathcal{M C}\left(M, \mathcal{H} / \mathcal{H}^{0}\right)_{-1}^{1}$, constructed via the KDPW scheme described in Section 2.2. will also be immersions into $U /\left(U_{\tau} \cap U_{\sigma}\right)$, for $\lambda \in \mathbb{R}^{*}$. Regular projections to $U / U_{\tau}$ and $U / U_{\sigma}$ will be discussed below.

\subsection{The Basic Equations Satisfied by $\mathfrak{G}_{-1}^{1}$-Compatible Immersions.} Let us return to the loop group defined in Section 3.1. Let $F$ be a local frame for an element of $\mathcal{M C}\left(M, \mathcal{H} / \mathcal{H}^{0}\right)_{-1}^{1}$, that is, $F \in \mathcal{M C}(\Sigma, \mathcal{H})_{-1}^{1}$, for some contractible open set $\Sigma$ containing the base point $x_{0} . F$ takes its values in $U:=G_{\rho}$ for $\lambda \in \mathbb{R}^{*}$, so, to simplify notation, we will always assume $\lambda \in \mathbb{R}^{*}$ in the following discussion. As in the introduction, let

$$
\mathfrak{u}=\mathfrak{k} \oplus \mathfrak{p}=\mathfrak{u}_{+} \oplus \mathfrak{u}_{-}
$$

be the canonical decompositions associated to $\tau$ and $\sigma$ respectively. We have the orthogonal (with respect to the Killing-form of $\mathfrak{g}$ ) decomposition

$$
\mathfrak{u}=\mathfrak{u}^{++} \oplus \mathfrak{u}^{+-} \oplus \mathfrak{u}^{-+} \oplus \mathfrak{u}^{--},
$$

where

$$
\begin{aligned}
\mathfrak{u}^{++}=\mathfrak{k} \cap \mathfrak{u}_{+}=: \mathfrak{k}^{\prime}, & \mathfrak{u}^{+-}=\mathfrak{k} \cap \mathfrak{u}_{-}, \\
\mathfrak{u}^{--}=\mathfrak{p} \cap \mathfrak{u}_{-}=: \mathfrak{p}^{\prime}, & \mathfrak{u}^{-+}=\mathfrak{p} \cap \mathfrak{u}_{+} .
\end{aligned}
$$

Let $\Omega(W)$ denote the set of real-valued 1-forms on a manifold $W$.

Lemma 3.3. $\quad$ (i) If $F$ is any element of $\mathcal{M C}(\Sigma, \mathcal{H})_{-1}^{1}$, then the MaurerCartan form $\alpha^{\lambda}=F^{-1} \mathrm{~d} F$ has the expansion

$$
\alpha^{\lambda}=\alpha_{0}^{++}+\alpha_{1}^{+-}\left(\lambda-\lambda^{-1}\right)+\alpha_{1}^{--}\left(\lambda+\lambda^{-1}\right),
$$

where

$$
\alpha_{0}^{++} \in \mathfrak{u}^{++} \otimes \Omega(M), \quad \alpha_{1}^{+-} \in \mathfrak{u}^{+-} \otimes \Omega(M), \quad \alpha_{1}^{--} \in \mathfrak{u}^{--} \otimes \Omega(M) .
$$


For any fixed value of $\lambda \in \mathbb{R}^{*}$, we have, in addition to the integrability of $\alpha$, the equation

$$
\mathrm{d} \alpha_{0}^{++}+\alpha_{0}^{++} \wedge \alpha_{0}^{++}=-4 \alpha_{1}^{--} \wedge \alpha_{1}^{--} .
$$

(ii) Conversely, suppose $\alpha^{\lambda}$ is a family of $\mathfrak{u}$-valued 1-forms on $\Sigma$, of the form (8), for $\lambda \in \mathbb{R}^{*}$, which is integrable for all $\lambda$. Then there exists a unique map $F \in \mathcal{M C}(\Sigma, \mathcal{H})_{-1}^{1}$ whose Maurer-Cartan form is $\alpha^{\lambda}$.

Proof. (i) By definition, $\alpha$ has the expansion

$$
\begin{array}{r}
\alpha^{\lambda}=\sum_{i=-1}^{1}\left(\alpha_{i}^{++}+\alpha_{i}^{+-}+\alpha_{i}^{-+}+\alpha_{i}^{--}\right) \lambda^{i}, \\
\alpha_{i}^{++} \in \mathfrak{u}^{++} \otimes \Omega(M), \quad \alpha_{i}^{+-} \in \mathfrak{u}^{+-} \otimes \Omega(M), \\
\alpha_{i}^{-+} \in \mathfrak{u}^{-+} \otimes \Omega(M), \quad \alpha_{i}^{--} \in \mathfrak{u}^{--} \otimes \Omega(M) .
\end{array}
$$

Now

$$
\tau \alpha^{\lambda}=\sum_{i=-1}^{1}\left(\alpha_{i}^{++}+\alpha_{i}^{+-}-\alpha_{i}^{-+}-\alpha_{i}^{--}\right)(-\lambda)^{-i},
$$

and $\alpha$ is fixed by $\tau$, from which it follows that

$$
\alpha^{\lambda}=\alpha_{0}^{++}+\alpha_{0}^{+-}+\left(\alpha_{1}^{++}+\alpha_{1}^{+-}\right)\left(\lambda-\lambda^{-1}\right)+\left(\alpha_{1}^{-+}+\alpha_{1}^{--}\right)\left(\lambda+\lambda^{-1}\right) \text {. }
$$

Then

$$
\sigma \alpha^{\lambda}=\alpha_{0}^{++}-\alpha_{0}^{+-}-\left(\alpha_{1}^{++}-\alpha_{1}^{+-}\right)\left(\lambda-\lambda^{-1}\right)-\left(\alpha_{1}^{-+}-\alpha_{1}^{--}\right)\left(\lambda+\lambda^{-1}\right) \text {, }
$$

from which we conclude that $\alpha$ has the form given by (8).

Now let us expand the integrability condition

$$
\mathrm{d} \alpha^{\lambda}+\alpha^{\lambda} \wedge \alpha^{\lambda}=0 .
$$

This equation must hold for all values of $\lambda$, and it is straightforward to verify that this is equivalent to the following five equations

$$
\begin{array}{r}
\mathrm{d} \alpha_{0}^{++}+\alpha_{0}^{++} \wedge \alpha_{0}^{++}+2\left(\alpha_{1}^{--} \wedge \alpha_{1}^{--}-\alpha_{1}^{+-} \wedge \alpha_{1}^{+-}\right)=0, \\
\mathrm{~d} \alpha_{1}^{+-}+\alpha_{0}^{++} \wedge \alpha_{1}^{+-}+\alpha_{1}^{+-} \wedge \alpha_{0}^{++}=0, \\
\mathrm{~d} \alpha_{1}^{--}+\alpha_{0}^{++} \wedge \alpha_{1}^{--}+\alpha_{1}^{--} \wedge \alpha_{0}^{++}=0, \\
\alpha_{1}^{+-} \wedge \alpha_{1}^{--}+\alpha_{1}^{--} \wedge \alpha_{1}^{+-}=0, \\
\alpha_{1}^{--} \wedge \alpha_{1}^{--}=-\alpha_{1}^{+-} \wedge \alpha_{1}^{+-} .
\end{array}
$$

Now, at a fixed value of $\lambda$, the first four of these equations are merely the decomposition of (10) into its $\mathfrak{u}^{++}, \mathfrak{u}^{+-}, \mathfrak{u}^{--}$and $\mathfrak{u}^{-+}$ components respectively, and so, pointwise in $\lambda$, they give no new conditions beyond integrability. The last, however, equation (15), does imply a further condition, and, given the equation (11), it is equivalent to equation (9) of the Lemma. 
(ii) The converse is just the standard fact that a $\mathfrak{g}$-valued 1-form which satisfies the integrability condition is the Maurer-Cartan form of a smooth map into a Lie group $G$, unique up to an initial condition. This is applied for every $\lambda$, and the uniqueness is given by the normalization at the base point $x_{0}$ of $M$.

\section{Interpretation of the Solutions}

Given an element $F \in \mathcal{M C}\left(M, \mathcal{H} / \mathcal{H}^{0}\right)_{-1}^{1}$, for real values of $\lambda, F_{\lambda}$ is a map $M \rightarrow U /\left(K \cap U_{+}\right)$, as explained in Section 3. We will interpret the projections to both $U / K$ and $U / U_{+}$, but first, some more details about reflective submanifolds.

4.1. Strongly Curvature Invariant Immersions Associated to Reflective Submanifolds of Symmetric Spaces. In this section we outline some well-known facts. More details can be found in [19].

4.1.1. Totally Geodesic Submanifolds of Symmetric Spaces. Let $P=U / K$ be a (not necessarily Riemannian) connected semisimple symmetric space, where $K=U_{\tau}$, and $\mathfrak{u}=\mathfrak{k} \oplus \mathfrak{p}$ is the canonical decomposition of the Lie algebra of $U$. There is a natural one-to-one correspondence between connected totally geodesic submanifolds $N$ through the origin 0 of $P$ and linear subspaces $\mathfrak{p}^{\prime}$ of $\mathfrak{p}$ which are closed under the Lie triple product, that is, $\left[\mathfrak{p}^{\prime},\left[\mathfrak{p}^{\prime}, \mathfrak{p}^{\prime}\right]\right] \subset \mathfrak{p}^{\prime}$. One way to construct the submanifold $N$ from $\mathfrak{p}^{\prime}$ is by setting $\mathfrak{k}^{\prime}:=\left[\mathfrak{p}^{\prime}, \mathfrak{p}^{\prime}\right], \mathfrak{u}^{\prime}:=\mathfrak{k}^{\prime}+\mathfrak{p}^{\prime}$, defining $U^{\prime}$ to be the connected Lie subgroup of $U$ generated by $\mathfrak{u}^{\prime}$, and setting $K^{\prime}:=U^{\prime} \cap K$. Then $N=U^{\prime} / K^{\prime}$, and the tangent space at the origin, $T_{0} N$, is $\mathfrak{p}^{\prime}$. The involution $\tau^{\prime}$ defining $K^{\prime}$ is given by $\tau^{\prime}:=\left.\tau\right|_{\mathfrak{u}^{\prime}}$. Conversely, given such a totally geodesic submanifold, one can take $U^{\prime}$ to be the largest subgroup of $U$ which leaves $N$ invariant, and set $K^{\prime}:=U^{\prime} \cap K$.

4.1.2. Reflective Submanifolds. Now suppose given a symmetric space $U / K$ as above, and suppose given another involution $\hat{\sigma}$ of $U$ which commutes with $\tau$. Let $\mathfrak{u}=\mathfrak{u}_{+} \oplus \mathfrak{u}_{-}$be the canonical decomposition associated to $\sigma$. Now set

$$
\mathfrak{p}^{\prime}:=\mathfrak{u}_{-} \cap \mathfrak{p},
$$

then $\left[\mathfrak{p}^{\prime},\left[\mathfrak{p}^{\prime}, \mathfrak{p}^{\prime}\right]\right] \subset \mathfrak{p}^{\prime}$, and so there is an associated symmetric subspace $\left(U^{\prime}, K^{\prime}, \tau^{\prime}\right)$ of $(U, K, \tau)$, with $\mathfrak{u}^{\prime}=\mathfrak{k}^{\prime}+\mathfrak{p}^{\prime}$, and a totally geodesic submanifold $N=U^{\prime} / K^{\prime}$ of $P$, as described above.

It will be convenient sometimes to choose a larger symmetric subalgebra than the one described in Section 4.1 .1 to represent $N$; namely, set

$$
\mathfrak{k}^{\prime}:=\mathfrak{k} \cap \mathfrak{u}_{+}, \quad \mathfrak{u}^{\prime}:=\mathfrak{k}^{\prime}+\mathfrak{p}^{\prime} .
$$

Clearly $\left[\mathfrak{k}^{\prime}, \mathfrak{k}^{\prime}\right] \subset \mathfrak{k}^{\prime}$ and $\left[\mathfrak{k}^{\prime}, \mathfrak{p}^{\prime}\right] \subset \mathfrak{p}^{\prime}$, so $\left(\mathfrak{u}^{\prime}, \mathfrak{k}^{\prime}, \tau^{\prime}\right)$ is a symmetric subalgebra of $(\mathfrak{u}, \mathfrak{k}, \tau)$, where $\tau^{\prime}:=\left.\tau\right|_{\mathfrak{u}^{\prime}}$, and we have a corresponding symmetric subspace $\left(U^{\prime}, K^{\prime}, \tau^{\prime}\right)$. Since a totally geodesic submanifold through the origin of $U / K$ 
is determined by its tangent space at the origin (in this case $\mathfrak{p}^{\prime}$ ) it follows that $U^{\prime} / K^{\prime}$ is the submanifold corresponding to $\mathfrak{p}^{\prime}$ described in Section 4.1.1.

Note that in the situation just described we have the orthogonal decomposition

$$
\mathfrak{p}=\mathfrak{p}^{\prime} \oplus \mathfrak{p}^{\perp}, \quad \mathfrak{p}^{\perp}=\mathfrak{p} \cap \mathfrak{u}_{+},
$$

and it is easy to check that $\mathfrak{p}^{\perp}$ is also closed under the Lie triple product. Thus $P$ is a reflective submanifold.

If $U / K$ is Riemannian then one can also show that, conversely, every reflective submanifold is associated canonically to a second involution $\sigma$ which commutes with $\tau$, and so may be characterized by the pairwise symmetric Lie algebra $(\mathfrak{u}, \tau, \sigma)$.

In the Riemannian case we also have the orthogonal decompositions

$$
\begin{aligned}
\mathfrak{k}=\left(\left[\mathfrak{p}^{\prime}, \mathfrak{p}^{\prime}\right] \cup\left[\mathfrak{p}^{\prime \perp}, \mathfrak{p}^{\prime \perp}\right]\right) & \oplus\left[\mathfrak{p}^{\prime}, \mathfrak{p}^{\prime \perp}\right], \\
\mathfrak{p} & =\mathfrak{p}^{\prime} \oplus \mathfrak{p}^{\prime \perp},
\end{aligned}
$$

and the relations

$$
\begin{aligned}
{\left[\left[\mathfrak{p}^{\prime}, \mathfrak{p}^{\prime}\right], \mathfrak{p}^{\prime \perp}\right] } & =\left[\left[\mathfrak{p}^{\prime}, \mathfrak{p}^{\prime \perp}\right], \mathfrak{p}^{\prime}\right] \subset \mathfrak{p}^{\perp} \\
{\left[\left[\mathfrak{p}^{\prime \perp}, \mathfrak{p}^{\prime \perp}\right], \mathfrak{p}^{\prime}\right] } & =\left[\left[\mathfrak{p}^{\prime}, \mathfrak{p}^{\perp \perp}\right], \mathfrak{p}^{\prime \perp}\right] \subset \mathfrak{p}^{\prime} .
\end{aligned}
$$

4.1.3. The $\mathfrak{G}_{\mathfrak{p}^{\prime}}$-Geometry. Any pairwise symmetric Lie algebra $(\mathfrak{u}, \tau, \sigma)$ induces a strongly curvature invariant Grassmann geometry, which we call the $\mathfrak{G}_{\mathfrak{p}^{\prime}}$-geometry, defined by the orbit of $\mathfrak{p}^{\prime}=\mathfrak{p} \cap \mathfrak{u}_{-}$under the action of $U$ on

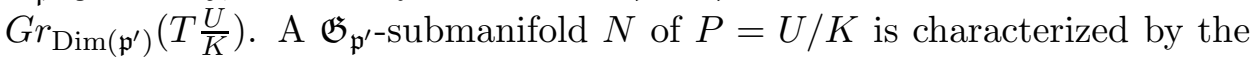
property that it is $\operatorname{Dim}\left(\mathfrak{p}^{\prime}\right)$-dimensional, and its tangent space at each point is $\mathfrak{p}^{\prime}$, if we identify the tangent bundle of $P$ with $\mathfrak{p}$ via left translation.

4.2. The Projection to $U / K$. Let $N=U / K$ be the reflective submanifold associated with the pairwise symmetric Lie algebra $(\mathfrak{u}, \tau, \sigma)$, and set $\mathfrak{p}^{\prime}=$ $\mathfrak{p} \cap \mathfrak{u}_{-}$as before. Set

$$
R_{\lambda}:=\frac{\left(\lambda+\lambda^{-1}\right)^{2}}{4} .
$$

For simplicity, we consider the spectral parameter in the interval $\mathbb{R}_{+}:=$ $(0, \infty) \in \mathbb{R}$. The following would analogously apply to the interval $(-\infty, 0)$. As $\lambda$ varies through $\mathbb{R}_{+}, R_{\lambda}$ varies through the interval $[1, \infty)$, achieving the value 1 only at $\lambda=1$.

Theorem 4.1. $\quad$ (i) Let $F$ be an element of $\mathcal{M C}\left(M, \mathcal{H} / \mathcal{H}^{0}\right)_{-1}^{1}$, where $M$ is simply connected and $\operatorname{Dim}(M)=\operatorname{Dim}\left(\mathfrak{p}^{\prime}\right)$. Let $f_{\lambda}: M \rightarrow P$ be the family of maps obtained by projecting $F_{\lambda}$, for $\lambda \in \mathbb{R}_{+}$, and assume that $f_{\lambda}$ is regular on $M$ for some value of $\lambda$. Then:

(a) $f_{\{\lambda=1\}}(M)$ is an open submanifold of the reflective submanifold $N \subset P$. 
(b) Let $M_{R}$ be the manifold $M$ with the pull-back metric induced from $f_{\{\lambda=1\}}: M \rightarrow N$, scaled by a factor $R>0$, so that $M_{R}$ is a open submanifold of $N_{R}$. Then, for other values of $\lambda \in \mathbb{R}_{+}$, $f_{\lambda}$ is an isometric normal curvature preserving $\mathfrak{G}_{\mathfrak{p}^{\prime}}$-immersion, $f_{\lambda}: M_{R_{\lambda}} \rightarrow P$.

(ii) Conversely, let $M$ be a simply connected, immersed, open submanifold of $N, R>1$, and suppose $f: M_{R} \rightarrow P$ is an isometric normal curvature preserving $\mathfrak{G}_{\mathfrak{p}^{\prime}}$-immersion. Choose $\lambda_{0} \in \mathbb{R}_{+}$such that $R=R_{\lambda_{0}}$. Then there exists an element $F \in \mathcal{M C}\left(M, \mathcal{H} / \mathcal{H}^{0}\right)_{-1}^{1}$ such that the projection of $F$ to $U / K$ at $\lambda=\lambda_{0}$ is $f$.

Proof. (i) The conditionis that need to be satisfied are essentially local, so we may as well assume $M$ is contractible and take a global frame for $F$, which we also denote by $F$. The left translation to the identity of the tangent space to the symmetric space $U / K$ is just $\mathfrak{p}$. Let $\langle\cdot, \cdot \cdot\rangle$ be the canonical metric on $\mathfrak{p}$, given by the Killing form. Choose a frame for $T_{x} M, e_{i}, i=1, \ldots, m$, which is orthonormal with respect to the pull-back metric. Then we have the projection to $\mathfrak{p}$ of $\alpha$,

$$
\pi_{\mathfrak{p}} F^{-1} \mathrm{~d} F=: \theta^{\lambda}=\sum_{i} \theta_{i}^{\lambda}
$$

where $\theta_{i}^{\lambda}\left(e_{i}\right)=: \hat{e}_{i}$ are orthonormal with respect to $\langle\cdot, \cdot\rangle$, that is, $\theta_{i}^{\lambda}$ are the coframe to $e_{i}$, with indices lowered. Comparing the definition of $\theta^{\lambda}$ with the form of $\alpha$ given by (8), we can also see that

$$
\theta^{\lambda}=\alpha_{1}^{--}\left(\lambda+\lambda^{-1}\right),
$$

which takes its values in $\mathfrak{p}^{\prime}$. It follows that $f_{\lambda}$ is a $\mathfrak{G}_{\mathfrak{p}^{\prime}}$-immersion.

Now the pullback metric is given by

$$
\mathrm{d} s_{\lambda}^{2}=\sum_{i}\left\langle\theta_{i}^{\lambda}, \theta_{i}^{\lambda}\right\rangle .
$$

Evidently, for any real value of $\lambda$, the metric is just a constant multiple of the metric for the immersion obtained at $\lambda=1$, that is:

$$
\mathrm{d} s_{\lambda}^{2}=\frac{\left(\lambda+\lambda^{-1}\right)^{2}}{4} \mathrm{~d} s_{1}^{2} .
$$

At $\lambda=1$, the Maurer-Cartan form of $F$ is:

$$
\alpha^{1}=\alpha_{0}^{++}+\theta^{1}
$$

which takes its values in $\mathfrak{u}^{\prime}=\mathfrak{k}^{\prime} \oplus \mathfrak{p}^{\prime}$. It follows that, at $\lambda=1, F$ is a frame for a map into the totally geodesic submanifold $U^{\prime} / K^{\prime}$, in other words, $f_{1}$ is an isometric immersion $M \rightarrow U^{\prime} / K^{\prime}$. For other values of $\lambda \in \mathbb{R}_{+}, f_{\lambda}$ is an isometric immersion $M_{R_{\lambda}} \rightarrow U / K$.

Finally, the connection 1-form for $f_{\lambda}$ is given by the projection to $\mathfrak{k}$ of the Maurer-Cartan form of $F_{\lambda}$, which, from (8) is

$$
\pi_{\mathfrak{k}} \alpha^{\lambda}=\alpha_{0}^{++}+\alpha_{1}^{+-}\left(\lambda-\lambda^{-1}\right),
$$


where $\alpha_{0}^{++} \in \mathfrak{k} \cap \mathfrak{u}_{+} \otimes \Omega(M)$ and $\alpha_{1}^{+-} \in \mathfrak{k} \cap \mathfrak{u}_{-} \otimes \Omega(M)$. We have the relations:

$$
\begin{aligned}
{\left[\mathfrak{k} \cap \mathfrak{u}_{+}, \mathfrak{p}^{\prime}\right] \subset \mathfrak{p}^{\prime}, } & {\left[\mathfrak{k} \cap \mathfrak{u}_{+}, \mathfrak{p}^{\prime \perp}\right] \subset \mathfrak{p}^{\prime \perp}, } \\
{\left[\mathfrak{k} \cap \mathfrak{u}_{-}, \mathfrak{p}^{\prime}\right] \subset \mathfrak{p}^{\prime \perp}, } & {\left[\mathfrak{k} \cap \mathfrak{u}_{-}, \mathfrak{p}^{\prime \perp}\right] \subset \mathfrak{p}^{\prime} . }
\end{aligned}
$$

It follows that the $\mathfrak{k}^{\prime}$ component of the connection, $\alpha_{0}^{++}$, is the sum of the connections on $\left.T M\right|_{M_{R_{\lambda}}}$ and $\left.T^{\perp} M\right|_{M_{R_{\lambda}}}$, whilst $\alpha_{1}^{+-}\left(\lambda-\lambda^{-1}\right)$ is the second fundamental form.

Fixing a choice of bases for $\mathfrak{p}^{\prime}$ and $\mathfrak{p}^{\prime}$ gives a bundle isomorphism between $\left.T M\right|_{M_{R_{\lambda}}}$ and $\left.T M\right|_{M}$ as well as between $\left.T^{\perp} M\right|_{M_{R_{\lambda}}}$ and $\left.T^{\perp} M\right|_{M}$. The sum of the tangential and normal connections do not depend on $\lambda$, so this 1-form pulls-back identically via this isomorphism. Since we already know that the tangent bundle-connection pairs are isomorphic (the metrics differ by a constant scalar multiple), it follows that the normal bundle-connection pairs are also isomorphic.

(ii) For the converse, we argue locally first, that is, choose a contractible subset $\Sigma_{R}$ of $M_{R}$, which contains the normalization point, so that a frame $F: \Sigma_{R} \rightarrow U$ exists for $f$. The Maurer-Cartan form of $F$ has the decomposition

$$
\begin{array}{r}
F^{-1} \mathrm{~d} F=\alpha^{++}+\alpha^{+-}+\alpha^{-+}+\alpha^{--}, \\
\alpha^{ \pm \pm} \in \mathfrak{u}^{ \pm \pm} \otimes \Omega(M) .
\end{array}
$$

The assumption that $f$ is a $\mathfrak{G}_{\mathfrak{p}^{\prime}}$-immersion means that $T^{\perp} \Sigma_{R}=$ $\left.\mathfrak{p}^{\perp}\right|_{\Sigma_{R}}$, where, as usual, we identify $T P$ with $\mathfrak{p}$. Hence the projection to $\mathfrak{p}^{\perp}$ of $F^{-1} \mathrm{~d} F$ is zero, that is, $\alpha^{-+}=0$. Now define a 1 -form

$$
\alpha^{\lambda}=\alpha^{++}+\left(\lambda-\lambda^{-1}\right) \frac{\alpha^{+-}}{\lambda_{0}-\lambda_{0}^{-1}}+\left(\lambda+\lambda^{-1}\right) \frac{\alpha^{--}}{\lambda_{0}+\lambda_{0}^{-1}} .
$$

By definition, $\alpha^{\lambda_{0}}=F^{-1} \mathrm{~d} F$ and is therefore integrable. Further, we saw in Lemma 3.3 that $\alpha^{\lambda}$ being integrable for all values of $\lambda$ is equivalent to integrability at a single value of $\lambda$ plus the equation

$$
\begin{aligned}
\mathrm{d} \alpha^{++}+\alpha^{++} \wedge \alpha^{++} & =-\frac{4}{\left(\lambda_{0}+\lambda_{0}\right)^{2}} \alpha^{--} \wedge \alpha^{--}, \\
& =-\frac{1}{R} \alpha^{--} \wedge \alpha^{--} .
\end{aligned}
$$

We also showed in Lemma 3.3 that $\left.\mathcal{M C}\left(\Sigma_{R}, \mathcal{H} / \mathcal{H}^{0}\right)\right|_{-1} ^{1}$ consists precisely of families, for $\lambda \in \mathbb{R}^{*}$, of equivalence classes of frames $F_{\lambda}$ : $\Sigma_{R} \rightarrow U$ whose Maurer-Cartan forms have the form (20) and satisfy (21), so we only need to show that this is satisfied.

The 1 -forms $\alpha^{--}$and $\alpha^{++}$have the same interpretation as in the first part of the proof. On the other hand, we also assumed that $M$ 
is an open immersed submanifold of the reflective submanifold $N$. If $g: M \rightarrow N \subset P$ is this immersion, and $G: \Sigma \rightarrow U$ a frame, where $\Sigma=\Sigma_{R}$ as a point set, then the Maurer-Cartan form is

$$
G^{-1} \mathrm{~d} G=\beta^{++}+\beta^{--},
$$

with $\beta^{ \pm \pm} \in \mathfrak{u}^{ \pm \pm} \otimes \Omega(M)$. There is no +- term, because $\Sigma$ is a totally geodesic submanifold of $P$, and, from the relation (19), the +- term of the Maurer-Cartan is the second fundamental form. Again, $\beta^{--}$ is the coframe for $g$, and $\beta^{++}$is the sum of the connections on $T \Sigma$ and $T^{\perp} \Sigma$.

Because $f$ is an isometric normal curvature preserving immersion, we can choose a bundle isomorphism $\phi: T \Sigma_{R} \oplus T^{\perp} \Sigma_{R} \rightarrow T \Sigma \oplus T^{\perp} \Sigma$, such that,

$$
\phi^{*} \beta^{++}=\alpha^{++}, \quad \sqrt{R} \phi^{*} \beta^{--}=\alpha^{--} .
$$

The factor of $\sqrt{R}$ is due to the fact that the metric on $\Sigma_{R}$ is $R$ times that on $\Sigma$. But then the $\mathfrak{k} \cap \mathfrak{u}_{+}$component of the integrability condition for $G^{-1} \mathrm{~d} G$ is

$$
\mathrm{d} \beta^{++}+\beta^{++} \wedge \beta^{++}=-\beta^{--} \wedge \beta^{--},
$$

and this, combined with (23) is equivalent to (21). Hence the 1form $\alpha^{\lambda}$ is integrable for all $\lambda$, and can be integrated on the simply connected set $\Sigma_{R}$ to obtain an element of $\left.\mathcal{M C}\left(\Sigma_{R}, \mathcal{H} / \mathcal{H}^{0}\right)\right|_{-1} ^{1}$ which has the required property.

For the global picture, one can show that, even though a global frame $F$ may not exist, the equivalence class in $\left.\mathcal{M C}\left(M, \mathcal{H} / \mathcal{H}^{0}\right)\right|_{-1} ^{1}$ is nevertheless well defined. This argument is given in an analogous situation in [5].

4.3. The Projection to $U / U_{+}$. We now consider the projection of an element of $\mathcal{M C}\left(M, \mathcal{H} / \mathcal{H}^{0}\right)_{-1}^{1}$, to $U / U_{+}$. We assume that $U, \tau, \sigma$ are as in Section 4.1

Proposition 4.1. Let $F \in \mathcal{M C}\left(M, \mathcal{H} / \mathcal{H}^{0}\right)_{-1}^{1}$. Let $F_{\lambda}: M \rightarrow U /\left(K \cap U_{+}\right)$ be the associated map, for $\lambda \in \mathbb{R}^{*}$. Then the map $\hat{f}_{\lambda}: M \rightarrow U / U_{+}$obtained by projection is a curved flat. Moreover, if the projection $f_{\lambda}: M \rightarrow U / K$ is regular then so is $\hat{f}_{\lambda}$.

Proof. Choosing a frame, $F \in \mathcal{M C}(\Sigma, \mathcal{H})_{-1}^{1}$, for $F$, on some contractible open set $\Sigma, F_{\lambda}$ is also a frame for $\hat{f}_{\lambda}$, and, we recall the expression for the Maurer-Cartan form $\alpha^{\lambda}=F_{\lambda}^{-1} \mathrm{~d} F_{\lambda}=\alpha_{0}^{++}+\alpha_{1}^{+-}\left(\lambda-\lambda^{-1}\right)+\alpha_{1}^{--}\left(\lambda+\lambda^{-1}\right)$, where the second two terms

$$
\alpha_{\mathfrak{u}_{-}}:=\alpha_{1}^{+-}\left(\lambda-\lambda^{-1}\right)+\alpha_{1}^{--}\left(\lambda+\lambda^{-1}\right),
$$

make up the $\mathfrak{u}_{-}$component of $\alpha$. Recall that $\hat{f}_{\lambda}$ is a curved flat if and only if $\alpha_{\mathfrak{u}_{-}} \wedge \alpha_{\mathfrak{u}_{-}}=0$. But this follows immediately from the conditions (14) and 
(15) obtained in Lemma 3.3. Finally, if $f_{\lambda}: M \rightarrow U / K$ is regular, then, as discussed above, the 1-form $\alpha_{1}^{--}$consists of $\operatorname{Dim}(M)$ linearly independent 1-forms. From (24), it follows that $\alpha_{\mathfrak{u}_{-}}$has the same property, and $\hat{f}_{\lambda}$ is also regular.

Corollary 4.2. Let $F \in \mathcal{M C}\left(M, \mathcal{H} / \mathcal{H}^{0}\right)_{-1}^{1}$, where $\operatorname{Dim}(M)=n$, and $U / U_{+}$ is Riemannian. Suppose that either of the associated maps $f_{\lambda}: M \rightarrow U / K$ or $\hat{f}_{\lambda}: M \rightarrow U / U_{+}$is regular for some (and hence all) values of $\lambda \in \mathbb{R}^{*}$. Then $M$ admits a topological covering by $\mathbb{R}^{n}$.

Proof. The map $\hat{f}_{\lambda}$ is a regular curved flat into a Riemannian symmetric space, which is intrinsically flat [12].

Combined with the converse part of Theorem 4.1 we obtain Corollary 1.2 ,

\section{Regularity of Projections to $U / K$}

We discussed, in Section 3, the local construction, via the KDPW method, of elements of $\mathcal{M C}\left(M, \mathcal{H} / \mathcal{H}^{0}\right)_{-1}^{1}$ which evaluate to immersions into $U /(K \cap$ $\left.U_{+}\right)$, provided $\operatorname{dim}(M) \leq \operatorname{rank}\left(U / U_{+}\right)$. Now we look at the regularity of projections to $U / K$.

Lemma 5.1. Suppose that the symmetric space $U / U_{+}$is Riemannian, and let $V$ be any vector subspace of $\mathfrak{u}_{-}$of dimension $n \leq r:=\operatorname{Rank}\left(U / U_{+}\right)$. Let $\pi_{V}: \mathfrak{u}_{-} \rightarrow V$ be the orthogonal projection with respect to the Killing metric on $\mathfrak{u}_{-}$. Then there exists a Cartan subalgebra $\mathfrak{m}$ of $\mathfrak{u}_{-}$such that

$$
\pi_{V} \mathfrak{m}=V .
$$

Moreover, the set of Cartan subalgebras of $\mathfrak{u}_{-}$which have this property is open and dense in the Grassmannian of Cartan subalgebras of $\mathfrak{u}_{-}$.

Proof. Let $\mathfrak{m}$ be any Cartan subalgebra of $\mathfrak{u}_{-}$, and choose an orthonormal basis $\left\{a_{1}, \ldots, a_{r}\right\}$ consisting of regular elements, ordered such that the projection $\pi_{V} \operatorname{Span}\left\{a_{1}, \ldots, a_{j}\right\}=\pi_{V} \mathfrak{m}=: V_{1}$, for some $j \leq r$. If the projection is not surjective, let $y$ be any non-zero element of $V \cap \mathfrak{m}^{\perp}$. Then, clearly $y \notin \mathfrak{m}$, so, since a Cartan subalgebra of $\mathfrak{u}_{-}$is equal to the centralizer of any of its regular elements, we must have $\left[a_{i}, y\right] \neq 0$ for any $i$. Then

$$
\left\langle\left[\left[a_{j+1}, y\right], a_{j+1}\right], y\right\rangle=\left\langle\left[a_{j+1}, y\right],\left[a_{j+1}, y\right]\right\rangle \neq 0,
$$

so the action of $\phi_{t}:=\operatorname{Ad}_{\exp \left(t\left[a_{j+1}, y\right]\right)}$ moves $a_{j+1}$ a non-zero amount in the direction of $y$, to first order in $t$. On the other hand, for all $i \neq j+1$, since $a_{k}$ are orthonormal, we have

$$
0=\left\langle a_{j+1}, a_{i}\right\rangle=\left\langle\left[a_{j+1}, y\right],\left[a_{i}, y\right]\right\rangle=\left\langle\left[\left[a_{j+1}, y\right], a_{i}\right], y\right\rangle,
$$

so $\phi_{t} a_{i}$ has no first order component in the direction of $y$. Choose $t$ small enough so that $\pi_{V} \operatorname{Span}\left\{\phi_{t} a_{1}, \ldots, \phi_{t} a_{j}\right\}$ still has dimension $j$. Then

$$
\pi_{V} \phi_{t} a_{j+1}=C t y+o(t)
$$


with $C \neq 0$, and, for $i \neq j+1$,

$$
\pi_{V} \phi_{t} a_{i}=\pi_{V} a_{i}+o(t) w+o\left(t^{2}\right) y,
$$

with $\langle w, y\rangle=0$, and $\left\langle\pi_{V} a_{i}, y\right\rangle=0$. It follows that $\pi_{V} \phi_{t} a_{j+1}$ is linearly independent from $\pi_{V} \phi_{t} a_{i}$. Hence $\pi_{V} \operatorname{Span}\left\{\phi_{t} a_{1}, \ldots, \phi_{t} a_{j+1}\right\}=: V_{2}$ has dimension $j+1$.

Now set $\mathfrak{m}_{2}:=\phi_{t} \mathfrak{m}$. This is still a Cartan subalgebra of $\mathfrak{u}_{-}$, and $\phi_{t} a_{i}$ are still regular elements, since the adjoint action of $U_{+}$preserves these properties. Hence, if $j+1<n$, we can repeat the above procedure, choosing an element $y_{1} \in V \cap \mathfrak{m}_{2}^{\perp}$. After $n-j$ steps, we have $\pi_{V} \mathfrak{m}_{n-j}=V$.

The above argument showed that there is a Cartan subalgebra which projects surjectively onto $V$ arbitrarily close to any Cartan subalgebra of $\mathfrak{m}$ of $\mathfrak{u}_{-}$. Since this projection property is also an open condition among Cartan subalgebras, this means that a generic Cartan subalgebra projects onto $V$.

Let $r:=\operatorname{Rank}\left(U / U_{+}\right)$. Regarding the next proposition, we first observe that a regular, $r$-dimensional, family of curved flats in $U / U_{+}$, given by $F_{+} \in \mathcal{M C}\left(\mathbb{R}^{r}, \Lambda G_{\sigma \rho}\right)_{1}^{1}$, certainly exists. One could take $F_{+}$to be the family associated to an embedding of a flat totally geodesic submanifold of $U / U_{+}$, for example. Further, if $U / U_{+}$is Riemannian, then the tangent space at each point to an $r$-dimensional regular curved flat is a Cartan subalgebra of $\mathfrak{u}_{-}[12$.

Proposition 5.1. Suppose that the symmetric space $U / U_{+}$is Riemannian and of rank $r$.

(i) If there exists a local solution $F \in \mathcal{M C}\left(\Sigma, \mathcal{H} / \mathcal{H}^{0}\right)_{-1}^{1}$ such that the associated maps $f_{\lambda}: \Sigma \rightarrow U / K$, for $\lambda \in \mathbb{R}^{*}$, are regular, then $n \leq r$.

(ii) Conversely, if $n \leq r$ then such a local regular solution can be constructed from any regular curved flat of dimension $r$, and the KDPW correspondence.

Proof. (i) First we examine the relationship between an element $F \in$ $\mathcal{M C}\left(\Sigma, \mathcal{H} / \mathcal{H}^{0}\right)_{-1}^{1}$ and the corresponding family of curved flats, $F_{+} \in$ $\mathcal{M C}\left(\Sigma, \Lambda G_{\sigma \rho}\right)_{1}^{1}$, obtained via the KDPW correspondence (44). They are related by the equation

$$
F=F_{+} G_{-},
$$

for some $G_{-} \in \mathcal{M C}\left(\Sigma, \Lambda G_{\sigma \rho}\right)_{-\infty}^{0}$. The Maurer-Cartan form of $F_{+}$ has the expansion

$$
F_{+}^{-1} \mathrm{~d} F_{+}=\psi \lambda
$$

for some $\psi \in \mathfrak{u}_{-} \otimes \Omega(\Sigma)$, and $G_{-}$has the expansion

$$
G_{-}=\sum_{-\infty}^{0} C_{i} \lambda^{i}, \quad C_{2 j} \in U_{+}, C_{2 j+1} \in U_{-} .
$$


Now

$$
\begin{aligned}
F^{-1} \mathrm{~d} F & =\alpha_{0}^{++}+\alpha_{1}^{+-}\left(\lambda-\lambda^{-1}\right)+\alpha_{1}^{--}\left(\lambda+\lambda^{-1}\right) \\
& =G_{-}^{-1}\left(F_{+}^{-1} \mathrm{~d} F_{+}\right) G_{-}+G_{-}^{-1} \mathrm{~d} G_{-} \\
& =C_{0}^{-1}(\psi \lambda) C_{0}+\sum_{i=-\infty}^{0} D_{i} \lambda^{i}
\end{aligned}
$$

where $D_{i}$ and $C_{0}$ do not depend on $\lambda$. As discussed above, the coframe for the projection to $U / K$ is obtained from the 1 -form $\alpha_{1}^{--}\left(\lambda+\lambda^{-1}\right)$, and so the condition for $f: \Sigma \rightarrow U / K$ to be an immersion for all $\lambda \in \mathbb{R}^{*}$ is that $\alpha_{1}^{--}$consists of $n$ linearly independent 1 -forms. From the above expansion of $F^{-1} \mathrm{~d} F$, we have

$$
\alpha_{1}^{--}=\pi_{\mathfrak{p}^{\prime}} \operatorname{Ad}_{C_{0}^{-1}} \psi
$$

Suppose first that we have a solution such that $f_{\lambda}$ is an immersion. Because $F_{+}$is a curved flat in $U / U_{+}$, it is necessarily tangent to a flat of $U / U_{+}$, which means that $\psi$ takes its values in an Abelian subalgebra $\mathfrak{a}_{1} \subset \mathfrak{u}_{-}$. Since the adjoint action of $U_{+}$on $\mathfrak{u}_{-}$takes Abelian subalgebras to Abelian subalgebras, $\mathrm{Ad}_{C_{0}^{-1}} \psi$ also takes it values in an Abelian subalgebra $\mathfrak{a} \subset \mathfrak{u}_{-}$. If $\pi_{\mathfrak{p}^{\prime}} \operatorname{Ad}_{C_{0}^{-1}} \psi$ consists of $n$ linearly independent 1 -forms, then it is necessary that $\pi_{\mathfrak{p}^{\prime}} \mathfrak{a}$ has dimension $n$, and so the rank of $U / U_{+}$is at least $n$.

(ii) Conversely, suppose that $n \leq r$. By Lemma 5.1, there exists a Cartan subalgebra $\mathfrak{a}$ of $\mathfrak{u}_{-}$such that $\pi_{\mathfrak{p}^{\prime}} \mathfrak{a}=\mathfrak{p}^{\prime}$. Take any curved flat family $F_{+} \in \mathcal{M C}\left(\Sigma, \Lambda G_{\sigma \rho}\right)_{1}^{1}$ which is regular, that is, $\left.\psi \lambda\right|_{x}=\left.F_{+}^{-1} \mathrm{~d} F_{+}\right|_{x}$ consists of $n$ linearly independent 1-forms and takes its values in a Cartan subalgebra $\mathfrak{m}_{x} \subset \mathfrak{u}_{-}$, for all $x$ in some neighbourhood of the initial condition $x_{0} \in \Sigma$.

We can assume that $x_{0}$ is the point at which all our loop group maps are normalized, that is, $F\left(x_{0}\right)=F_{+}\left(x_{0}\right)=G_{-}\left(x_{0}\right)=I$ for all $\lambda$, where $\left.F \in \mathcal{M C}\left(\Sigma, \mathcal{H} / \mathcal{H}^{0}\right)\right|_{-1} ^{1}$ is obtained via the KDPW correspondence (4). We can also assume, after an action by $U_{+}$, that $\mathfrak{m}_{x_{0}}=\mathfrak{a}$, since $\mathfrak{a}$ is Cartan and $U_{+}$acts transitively on the Cartan subalgebras in $\mathfrak{u}_{-}$. At the normalization point $x_{0}$, equation (25) reduces to $\left.\alpha_{1}^{--}\right|_{x_{0}}=\left.\pi_{\mathfrak{p}^{\prime}} \psi\right|_{x_{0}}$. Since, $\pi_{\mathfrak{p}^{\prime}} \mathfrak{a}=\mathfrak{p}^{\prime}$, and $\mathfrak{a}$ and $\mathfrak{p}^{\prime}$ have the same dimension, it follows that $\left.\alpha_{1}^{--}\right|_{x_{0}}$ also consists of $n$ linearly independent 1-forms. This is an open condition, and hence the solution is an immersion on some neighbourhood of $x_{0}$.

Combining Theorem 4.1 with Proposition 5.1 we obtain Theorem 1.3 , 


\section{The Solutions Associated to Reflective Submanifolds of Compact Symmetric Spaces}

In this section we determine the answer to Problem 1.1 for the case that $U / K$ is a compact, simply connected, irreducible symmetric space. Clearly, we need only consider strongly curvature invariant $\mathfrak{G}$-geometries which admit non-totally geodesic submanifolds, so it is enough to go through Naitoh's list, given in the introduction.

6.1. The Geometry of $k$-Dimensional Submanifolds of the Sphere $S^{n}, 0<k<n$. The reflective submanifolds of the sphere $S^{n}$ are just the totally geodesic submanifolds, namely spheres of dimension $k<n$. For these, the normal bundle is flat, so isometric normal curvature preserving $\mathfrak{G}_{\mathfrak{p}^{\prime}}$-immersions of $N_{R}$ will be isometric immersions with flat normal bundle of a sphere of radius $\sqrt{R}$.

To check whether solutions exist, set $\mathfrak{u}=\mathfrak{s o}(n+1), \tau=\operatorname{diag}\left(I_{n},-1\right)$, where $I_{j}$ denotes the $j \times j$ identity matrix, $\sigma=\operatorname{diag}\left(I_{k},-I_{n+1-k}\right)$. Then $\mathfrak{k}=\mathfrak{s o}(n), \mathfrak{u}_{+}=\mathfrak{s o}(k) \times \mathfrak{s o}(n+1-k)$, and

$$
\mathfrak{p}^{\prime}=\mathfrak{p} \cap \mathfrak{u}_{-}=\left[\begin{array}{ccc}
0 & 0 & *_{k \times 1} \\
0 & 0 & 0 \\
*_{1 \times k} & 0 & 0
\end{array}\right],
$$

where $*_{i \times j}$ indicates an $i \times j$ submatrix. To identify the associated totally geodesic submanifold $N$, we can just look at $\mathfrak{p}^{\prime}$, which must be the tangent space to the origin. We can take $\mathfrak{k}^{\prime}=\left[\mathfrak{p}^{\prime}, \mathfrak{p}^{\prime}\right]$, and we see that $N=S O(k+$ $1) / S O(k)=S^{k}$. The rank of $U / U_{+}$is $\min (k, n+1-k)$, so, by Theorem 1.3. we need $k \leq n+1-k$. In other word, solutions exist if and only if $k \leq(n+1) / 2$.

\subsection{The Geometry of $k$-Dimensional Complex Submanifolds of the} Complex Projective Space $\mathbb{C} P^{n}, 0<k<n$. This is the analogue of the case of the sphere, substituting $\mathfrak{s u}(n+1)$ for $\mathfrak{s o}(n+1)$. In this case, $N=$ $S U(k+1) / S(U(k) \times U(1))$ and $U / U_{+}=S U(n+1) /(S(U(k) \times U(n+1-k))$. The dimension of $N$ is $2 k$, and the rank of $U / U_{+}$is $\min (k, n+1-k)$ so there is no solution for any $k$ or $n$.

\subsection{The Geometry of $n$-Dimensional Totally Real Submanifolds of}

$\mathbb{C} P^{n}$. This is the case $\mathfrak{u}=\mathfrak{s u}(n+1), \mathfrak{k}=\mathfrak{s}(\mathfrak{u}(n) \times \mathfrak{u}(1)), \mathfrak{u}_{+}=\mathfrak{s o}(n+1)$. Then $U / K=\mathbb{R} P^{n}$, and has dimension $n$, and $U / U_{+}$has rank $n$. Therefore, solutions exist for every $n$. The $\mathfrak{G}_{\mathfrak{p}^{\prime}}$-immersions are totally real isometric immersions of $S_{R}^{n}$ into $\mathbb{C} P^{n}$. In this case, the normal bundle is isomorphic to the tangent bundle via the complex structure, so the requirement that the immersion be normal curvature preserving is contained in the totally real condition.

It may be of interest to see the details explicitly: consider $S^{2 n+1}$ as the unit sphere in $\mathbb{C}^{n+1}$, with the Sasakian structure on $T S^{2 n+1}$ induced by multiplication by $i \in \mathbb{C}^{n+1}$, which we denote by $J$. We will call a submanifold 
$M$ of $S^{2 n+1}$ totally real if it is totally real as a submanifold of $\mathbb{C}^{n+1}$, which means $J T_{x} M \subset T_{x}^{\perp} M \oplus \mathbb{R} x$ for all $x$.

The Hopf fibration, $\pi: S^{2 n+1} \rightarrow \mathbb{C} P^{n}$, is given by the projection to $S^{2 n+1} / U(1)=\mathbb{C} P^{n}$, where the action of $U(1)$ is by following the integral curves of the vector field $J x$, for $x \in S^{2 n+1}$. The vertical space at $x$ to the fibration is given by the line through the origin in $T_{x} S^{2 n+1}$ spanned by $J x$. If $M=M^{n}$ is an $n$-dimensional immersed submanifold of $S^{2 n+1}$ which is transverse to the Hopf fibration for all $x$, then the projection to $\mathbb{C} P^{n}, \pi(M)$ is also an immersed submanifold of the same dimension. If $M$ is totally real in $S^{2 n+1}$, then $\pi(M)$ is also totally real (or Lagrangian) in $\mathbb{C} P^{n}$, that is the complex structure on the tangent bundle of $\mathbb{C} P^{n}$, which we also denote by $J$, takes the tangent space $T_{\pi(x)} \pi(M)$ isomorphically to its orthogonal complement $T_{\pi(x)}^{\perp} \pi(M)$. This follows from the fact that $J$ is induced from the complex structure on $\mathbb{C}^{n+1}$. Conversely, one can show, [29], that if $M$ is simply connected and $\hat{f}: M \rightarrow \mathbb{C} P^{n}$ is a Lagrangian immersion then there exists a lift to a Legendrian, (a special case of our definition of totally real) immersion $f: M \rightarrow S^{2 n+1}$ such that $\pi f=\hat{f}$.

Let $U=S U(n+1)$, represented by the matrix subgroup of $S O(2 n+2)$ consisting of all matrices of the form $\left[\begin{array}{cc}A & -B \\ B & A\end{array}\right]$, where $A$ and $B$ are $(n+$ $1) \times(n+1)$, and such that $\operatorname{det}(A+i B)=1$. The Lie algebra $\mathfrak{u}=s u(n+1)$ is thus represented as the subalgebra of $s o(2 n+2)$ consisting of matrices $\left[\begin{array}{cc}a & b \\ -b & a\end{array}\right]$, where $a$ is skew symmetric, $b$ is symmetric and $\operatorname{Tr}(b)=0$.

For a matrix $F \in U$, we regard the columns of $F$ as vectors $[a, b]^{t}=$ $a+b i \in \mathbb{R}^{2 n+2}=\mathbb{C}^{n+1}$. Then $F=[X, J X]$, where $J=\left[\begin{array}{cc}0 & -I_{n+1} \\ I_{n+1} & 0\end{array}\right]$ is the complex structure on $\mathbb{C}^{n+1}$. An adapted frame for a totally real immersion $f: M^{n} \rightarrow S^{2 n+1}$ is a map $F: M \rightarrow U$ given by

$$
F=[X, f, J X, J f]
$$

where the $n$ columns of $X$ are all in the normal space to the immersion. Thus the tangent space is contained in the span of the vectors making up $J X$ and $J f$.

Let $U=S U(n+1)$ as above. Let $\sigma=\operatorname{Ad}_{P}$, where $P=\operatorname{diag}\left(I_{n+1},-I_{n+1}\right)$, and $\tau=\operatorname{Ad}_{Q}$, for $Q=\operatorname{diag}\left(I_{n},-1, I_{n},-1\right)$. Then $U_{\sigma}$ and $U_{\tau}$ are isomorphic to $S O(n+1)$ and $S\left(U_{n} \times U_{1}\right)$ respectively.

Let $G=U^{\mathbb{C}}$ and, as before, define $\sigma, \tau$ and $\rho$ by the extensions (6) and (7), and the extension (5) for the complex conjugation on $G$, and set $\mathcal{H}=\Lambda G_{\rho \sigma \tau}$. Let $F$ be an element of $\mathcal{M C}\left(M, \mathcal{H} / \mathcal{H}^{0}\right)_{-1}^{1}$. Now $\mathcal{H}^{0}=\mathfrak{k}^{\prime}=\mathfrak{k} \cap \mathfrak{u}_{+}$consists of matrices in $s u(n+1)$ of the form $\operatorname{diag}\left(*_{n \times n}, *_{1 \times 1}, *_{n \times n}, *_{1 \times 1}\right)$, and so right multiplication by $\mathcal{H}^{0}$ fixes the $(n+1)^{\prime}$ 'th and last columns of $F$. We will therefore regard the $(n+1)$ 'th column of $F$ as a map $f$ into $S^{2 n+1}$. The 
Maurer-Cartan form of $F$ has the expression

$$
F^{-1} \mathrm{~d} F=\left[\begin{array}{cccc}
\omega & 0 & -\left(\lambda-\lambda^{-1}\right) \beta & -\left(\lambda+\lambda^{-1}\right) \theta \\
0 & 0 & -(\lambda+\lambda)^{-1} \theta^{t} & -\left(\lambda-\lambda^{-1}\right) \alpha \\
\left(\lambda-\lambda^{-1}\right) \beta^{t} & \left(\lambda+\lambda^{-1}\right) \theta & \omega & 0 \\
\left(\lambda+\lambda^{-1}\right) \theta^{t} & \left(\lambda-\lambda^{-1}\right) \alpha & 0 & 0
\end{array}\right]
$$

where $\omega$ and $\beta$ are an $n \times n$ matrix-valued 1-forms, $\theta$ is $n \times 1$ and $\alpha$ is $1 \times 1$. The condition (9) in this case reduces to the equation

$$
\mathrm{d} \omega+\omega \wedge \omega=4 \theta \wedge \theta^{t},
$$

which we shall return to below.

If we write $F=[X, f, J X, J f]$, then we see from (27) that $X^{t} \mathrm{~d} f=0$, and so the columns of $X$ are all contained in the normal space to the image of $f$. Thus, $f$ is totally real. The coframe for $f$ is given by the column vector

$$
[J X, J f]^{t} \mathrm{~d} f=\left[\left(\lambda+\lambda^{-1}\right) \theta^{t},\left(\lambda-\lambda^{-1}\right) \alpha\right]^{t},
$$

and so the condition that $f$ be an immersion is that the 1-forms which make up the components of this matrix span a space of dimension $n$.

At $\lambda=1$, we have $(J f)^{t} \mathrm{~d} f=\left(\lambda-\lambda^{-1}\right) \alpha=0$, so $J f$ is normal, and the immersion is Legendrian. At this point, the second fundamental form is

$$
[X, J f]^{t} \mathrm{~d}(J X)=\left[-\left(\lambda-\lambda^{-1}\right) \beta, 0\right]^{t},
$$

and this is also zero, so the immersion is totally geodesic Legendrian at $\lambda=1$. This projects to a totally geodesic Lagrangian immersion in $\mathbb{C} P^{n}$.

For other values of $\lambda, f$ is not horizontal, but, if we assume that the vector $\theta^{t}$ consists of $n$ linearly independent 1 -forms, then $f$ is transverse to the fibre of the Hopf projection. Thus $\pi \circ f$ is still a Lagrangian immersion into $\mathbb{C} P^{n}$. Writing $X=\left[X_{1}, \ldots, X_{n}\right]$, then, since $X_{i}$ are horizontal to $\pi$, and normal to the image of $f$, it follows that $\pi_{*} X_{i}$ are an orthonormal basis for the normal space to $\pi f$, and, therefore, $\pi_{*} J X_{i}=J \pi_{*} X_{i}$ are an orthonormal basis for the tangent space.

Let $e_{i}$ be the vector fields on $M$ such that $\pi_{*} J X_{i}=\pi_{*} \circ f_{*} e_{i}$. Then $f_{*} e_{i}=J X_{i}+\left(f_{*} e_{i}\right)^{\perp}$, where the last term is in the vertical space. Hence $J X_{i}^{t} \mathrm{~d} f\left(e_{j}\right)=\delta_{i j}$, and so the coframe for the orthonormal (with respect to the metric induced by $\pi \circ f$ ) basis $e_{i}$ is given by

$$
\theta_{\lambda}:=(J X)^{t} \mathrm{~d} f=\left(\lambda+\lambda^{-1}\right) \theta .
$$

The connection induced by $\pi \circ f$ on the tangent space is given by $\omega=$ $(J X)^{t} \mathrm{~d}(J X)$, and thus the equation (28), which we can write as

$$
\mathrm{d} \omega+\omega \wedge \omega=\frac{4}{\left(\lambda+\lambda^{-1}\right)^{2}} \theta_{\lambda} \wedge \theta_{\lambda}^{t}
$$

is the statement that the induced curvature is constant and equal to $c_{\lambda}=$ $\frac{4}{\left(\lambda+\lambda^{-1}\right)^{2}}$. Equivalently, $\pi \circ f$ is an isometric immersion of a piece of a sphere of radius $\sqrt{R_{\lambda}}$. 
6.4. The Geometry of $2 n$-Real-Dimensional Totally Complex Submanifolds of the Quaternionic Projective Space $\mathbb{H} P^{n}$. The case $n=1$ is a 4 -sphere of constant curvature 4 , covered above, so we consider $n \geq 2$. In this case, $\mathfrak{u}=\mathfrak{s p}(n+1), \mathfrak{k}=\mathfrak{s p}(n) \times \mathfrak{s p}(1), \mathfrak{u}_{+}=\mathfrak{u}(n+1)$, and the reflective submanifold is $\mathbb{C} P^{n}$, which has dimension $2 n$. The rank of $U / U_{+}$ is $n+1$, so we need $2 n \leq n+1$, and solutions do not exist for $n>1$.

6.5. The Geometries Associated to Symmetric $R$-Spaces. Naitoh proved that the strongly curvature $\mathfrak{G}$-geometries in compact symmetric spaces associated to symmetric $R$-spaces only admit non-totally geodesic $\mathfrak{G}$-submanifolds which are homothetic to the corresponding reflective submanifold, with a factor $R<1$, opposite to what we seek. The analogue for the non-compact case, with $R>1$, was proved by Berndt et al. in [4] (where both cases are discussed). Hence we do not need to consider these cases.

Taking into account Corollary 1.2, this completes the proof of Theorem 1.4 .

\section{The Non-Compact Case}

Here we want to consider the case $R<1$. We start with the observation that the arguments given on the interpretation of the solutions of $\mathcal{M C}\left(M, \mathcal{H} / \mathcal{H}^{0}\right)_{-1}^{1}$ as projections to $U / K$ did not depend on what kind of conjugation $\rho$ we used for the reality condition. The reality condition only determined which subset of $\mathbb{C}^{*}$ corresponded to solutions in $U$, and hence the range of values for $R_{\lambda}=\frac{\left(\lambda+\lambda^{-1}\right)^{2}}{4}$.

We therefore consider now an alternative reality condition defined by the conjugation

$$
\left(\rho_{2} X\right)(\lambda):=\rho(X(1 / \bar{\lambda})) .
$$

Elements of $\Lambda G_{\rho_{2}}$ are $U$-valued for values of $\lambda$ in $S^{1}$, and it is easy to check the following

Lemma 7.1. Let $\mathcal{H}_{2}:=\Lambda G_{\tau, \sigma, \rho_{2}}$, where $\sigma$ and $\tau$ are as before. Then Theorem 4.1 also holds for $\mathcal{M C}\left(M, \frac{\mathcal{H}_{2}}{\mathcal{H}_{2}^{0}}\right)_{-1}^{1}$, replacing the statement $\lambda \in \mathbb{R}_{+}$with $\lambda \in S^{1} \backslash\{ \pm i\}$.

The values $\lambda= \pm i$ are excluded because the coframe $\theta^{\lambda}$ vanishes there. Note that the difference here is that for $\lambda \in S^{1}, R_{\lambda}$ takes its values in $(0,1)$, and this is the case we are interested in here.

Now define another reality condition $\tilde{\rho}$ by the involution:

$$
(\tilde{\rho} X)(\lambda):=\rho \circ \tau \circ \sigma(X(\bar{\lambda})) .
$$

Then $X$ is fixed by $\tau, \sigma$ and $\rho_{2}$ if and only if $X$ is fixed by $\tau, \sigma$ and $\tilde{\rho}$. Hence

$$
\Lambda G_{\tau \sigma \rho_{2}}=\Lambda G_{\tau \sigma \tilde{\rho}}
$$

and thus the loop group is exactly the type we have already considered, since $\tilde{\rho}$ is of the form (5). Thus we have the following 
Lemma 7.2. Let $\mathcal{H}_{2}:=\Lambda G_{\tau \sigma \rho_{2}}$, as in Lemma 7.1. Then Theorem 4.1 also holds for $\mathcal{M C}\left(M, \frac{\mathcal{H}_{2}}{\mathcal{H}_{2}^{0}}\right)_{-1}^{1}$, for $\lambda \in \mathbb{R}_{+}$, replacing the pairwise symmetric Lie algebra $(\mathfrak{u}, \tau, \sigma)$ with $(\tilde{\mathfrak{u}}, \tau, \sigma)$, where $\tilde{\mathfrak{u}}$ is the real form of the Lie algebra $\mathfrak{g}$ defined by

$$
\tilde{\rho}:=\rho \circ \tau \circ \sigma .
$$

Hence the case $R<1$ is really just the case $R>1$ for a symmetric space associated to a different real form of $G$, and so we can use our previous analysis to discuss the problem of existence of solutions. Specifically, the projection $f_{\lambda}: M \rightarrow U / K$ has solutions for $\lambda \in S^{1}$ if and only if the projection $\tilde{f}: M \rightarrow \widetilde{U} / \widetilde{K}$ has solutions for $\lambda \in \mathbb{R}^{*}$. Set $\widetilde{U}_{+}:=\widetilde{U}_{\sigma}$. The point that we will use is: if $\widetilde{U} / \widetilde{U}_{+}$is Riemannian then solutions exist if and only if $\operatorname{Dim}(U / K)=\operatorname{Rank}\left(\widetilde{U} / \widetilde{U}_{+}\right)$.

\subsection{The Geometry of $k$-Dimensional Submanifolds of the Real Hy-} perbolic Space. This is the analogue of the case of the sphere. Normal curvature preserving $\mathfrak{G}_{\mathfrak{p}^{\prime}}$-immersions of $N_{R}$ will be isometric immersions with flat normal bundle of a hyperbolic space of radius $\sqrt{R}$. We have the analogous construction with $\mathfrak{u}=\mathfrak{s o}(n, 1), \tau=\operatorname{Ad}_{Q}, Q=\operatorname{diag}\left(I_{n},-1\right)$, $\sigma=\operatorname{Ad}_{P}, P=\operatorname{diag}\left(I_{k},-I_{n+1-k}\right)$.

Set $J_{p, q}=\operatorname{diag}\left(I_{p},-I_{q}\right)$, and define

$$
\begin{array}{r}
S O(p, q, \mathbb{C})=\left\{X \in G L(p+q, \mathbb{C}) \mid X^{t} J_{p, q} X=J\right\}, \\
S O(p, q)=\{X \in S O(p, q, \mathbb{C}) \mid \bar{X}=X\} .
\end{array}
$$

According to the argument presented above, if we replace the involution $\rho X=\bar{X}$ with

$$
\tilde{\rho} X=\overline{\tau \sigma X}
$$

then we can analyze the corresponding objects in the real form given by $\widetilde{U}=$ $S O(n, 1, \mathbb{C})_{\tilde{\rho}}$ for existence of solutions. Let $\phi: G L(n+1, \mathbb{C}) \rightarrow G L(n+1, \mathbb{C})$ be defined by $\phi=A d_{T}$, for $T=\operatorname{diag}\left(I_{k}, i I_{n}, 1\right)$. Then it is easy to check that

$$
\phi: S O(n, 1, \mathbb{C})_{\tilde{\rho}} \rightarrow S O(k, n+1-k)
$$

is an isomorphism. $\phi$ commutes with $\sigma$, so we have

$$
\tilde{\mathfrak{u}}=\mathfrak{s o}(k, n+1-k), \quad \tilde{\mathfrak{u}}_{+}=\mathfrak{s o}(k) \times \mathfrak{s o}(n+1-k),
$$

which is compact. Hence $\widetilde{U} / \widetilde{U}_{+}$is Riemannian, and of $\operatorname{rank} \min (k, n+1-k)$, and we can say that solutions exist if and only if $k \leq(n+1) / 2$.

Remark 7.3. The solutions $\tilde{f}_{\lambda}: M \rightarrow \widetilde{U} / \widetilde{K}$ here, for $\lambda \in \mathbb{R}^{*}$, are actually strongly curvature invariant $\mathfrak{G}$-immersions, $H_{R}^{k} \rightarrow S O(k, n+1-$ $k) / S O(k, n-k)$, and do not appear in Naitoh's list because the target space is non-Riemannian. 
7.2. The Geometry of Complex Submanifolds of Complex Hyperbolic Space $\mathbb{C} H^{n}$. This is the analogue of the previous case, substituting $\mathfrak{s u}(n, 1)$ for $\mathfrak{s o}(n, 1)$. Then $\tilde{\mathfrak{u}}=\mathfrak{s u}(k, n+1-k)$, and $\tilde{\mathfrak{u}}_{+}=\mathfrak{s}(\mathfrak{u}(k) \times \mathfrak{u}(n+1-k))$, so $\widetilde{U} / \widetilde{U}_{+}$is Riemannian, and, as in the compact analogue, there is no solution for any $k$ or $n$.

\subsection{The Geometry of $n$-Dimensional Totally Real Submanifolds of} $\mathbb{C} H^{n}$. Here $\mathfrak{u}=\mathfrak{s u}(n, 1), \mathfrak{k}=\mathfrak{s}(\mathfrak{u}(n) \times \mathfrak{u}(1)), \mathfrak{u}_{+}=\mathfrak{s} \mathfrak{o}(n, 1)$. The complex Lie algebra is $\mathfrak{g}=\mathfrak{s l}(n+1, \mathbb{C})$, and the involutions on $\mathfrak{g}$ are given by $\rho x=$ $\operatorname{Ad}_{J}\left(\bar{x}^{t}\right)^{-1}, \tau x=\operatorname{Ad}_{J} x$ and $\sigma x=\operatorname{Ad}_{J}\left(x^{t}\right)^{-1}$, where $J=\operatorname{diag}\left(I_{n},-1\right)$.

Setting $\tilde{\rho}(x)=\rho \circ \tau \circ \sigma(x)=\operatorname{Ad}_{J} \bar{x}$, we obtain

$$
\tilde{\mathfrak{u}}=\mathfrak{s l}(n+1, \mathbb{R}), \quad \tilde{\mathfrak{u}}_{+}=\mathfrak{s o}(n, 1) .
$$

In this case, $\tilde{\mathfrak{u}}_{+}$is not compact, so $\widetilde{U} / \widetilde{U}_{+}$is non-Riemannian. However, we do not want to show non-existence here, so this is no problem. To show that a local solution can be constructed from a curved flat in $\widetilde{U} / \widetilde{U}_{+}$, via the KDPW scheme, examining the converse part of the proof of Proposition 5.1, it will be enough to find an Abelian subalgebra $\mathfrak{a}$ in $\tilde{\mathfrak{u}}_{-}$which projects onto $\tilde{\mathfrak{p}} \cap \tilde{\mathfrak{u}}_{-}$. Then exponentiating $\mathfrak{a}$ will give a suitable curved flat to locally construct a regular solution, as in the proof of Proposition 5.1. The relevant subspaces consist of the matrices in $\mathfrak{s l}(n+1, \mathbb{R})$ as follows:

$$
\tilde{\mathfrak{u}}_{-}=\left\{\left[\begin{array}{cc}
A & b \\
-b^{t} & c
\end{array}\right]\right\}, \quad \tilde{\mathfrak{p}} \cap \tilde{\mathfrak{u}}_{-}\left\{\left[\begin{array}{cc}
0 & *_{n \times 1} \\
*_{1 \times n} & 0
\end{array}\right]\right\},
$$

where $A$ is a symmetric $n \times n$ matrix. Let $E_{i}$ be the $(n+1) \times(n+1)$ matrix whose $i$ 'th row is $[0, \ldots, 0,1,1]$ and $i$ 'th column is $[0, \ldots, 0,1,-1]$, and all other components are zero. Let $\hat{E}$ be the matrix whose $n$ 'th row is $[0, \ldots, 0,1,1]$, and whose $(n+1)$ 'st row is $[0, \ldots, 0,-1,-1]$, with all other components zero. Then $\left\{E_{1}, \ldots, E_{n-1}, \hat{E}\right\}$ generate such an Abelian subalgebra. Hence, a solution exists for every $n$.

7.4. The Geometry of $2 n$-Real-Dimensional Totally Complex Submanifolds of the Quaternionic Hyperbolic Space $\mathbb{H} H^{n}$. In this case, $\mathfrak{g}=\mathfrak{s p}(n+1, \mathbb{C}), \mathfrak{u}=\mathfrak{s p}(n, 1), \mathfrak{k}=\mathfrak{s p}(n) \times \mathfrak{s p}(1), \mathfrak{u}_{+}=\mathfrak{u}(n, 1)$, and the reflective submanifold is $\mathbb{C} H^{n}$, which has dimension $2 n$.

The involutions are $\rho X=\operatorname{Ad}_{K} \overline{\left(X^{t}\right)^{-1}}$, for $K=\operatorname{diag}\left(I_{n},-1, I_{n},-1\right)$, $\tau X=\operatorname{Ad}_{K} X, \sigma X=\operatorname{Ad}_{J} X$, for $J=\left[\begin{array}{cc}0 & I_{n+1} \\ -I_{n+1} & 0\end{array}\right]$. Setting $\tilde{\rho}(X)=$ $\rho \circ \tau \circ \sigma(X)=\operatorname{Ad}_{J} \overline{\left(X^{t}\right)^{-1}}=\bar{X}$, we have

$$
\tilde{\mathfrak{u}}=\mathfrak{s p}(n+1, \mathbb{R}), \quad \check{\mathfrak{k}}=\mathfrak{u}(n+1) .
$$

Now $\widetilde{U} / \widetilde{U}_{+}$is Riemannian and of rank $n+1$, hence, as in the compact case, we need $2 n \leq n+1$, so solutions do not exist for $n>1$.

Together with the remarks of Section 6.5, this completes the proof of Theorem 1.5. 


\section{Projections to Other Homogeneous Spaces}

There are many more reflective submanifolds besides those discussed in Sections [6] and 7. In all other (Riemannian) cases, the rank $r$ of $U / U_{+}$ must be less than the dimension of $\mathfrak{p}^{\prime}$, according to the results of Naitoh. However, we can still produce solutions of dimension $r$, and these correspond, under the projection to $U / K$ to $s u b-\mathfrak{G}_{\mathfrak{p}^{\prime}}$-immersions. In this case, they have the property that the connections on the natural bundles $\mathfrak{p}^{\prime}$ and $\mathfrak{p}^{\perp}$ are preserved, as $\lambda$ varies, rather than the tangent and normal bundles.

It may be be interesting to take projections to other homogeneous spaces $U / H$ where $H$ is a subgroup containing $K \cap U_{+}$, and we consider one example here.

\subsection{Reflective Submanifolds of Symmetric Spaces with Isometry} Group $G_{2}$. According to the classification in [19], the only reflective submanifolds associated to $G_{2}$ are the reflective submanifolds $S O(4)$ and $G_{2} / S O(4)$ in $G_{2}$, and $(S U(2) / S O(2)) \times(S U(2) / S O(2))$ in $G_{2} / S O(4)$. All of these have dimension greater than 2 , but the rank of $G_{2}$ is 2 , and so the rank of $G_{2} /\left(G_{2}\right)_{+}$will always be too small for the existence of local normal curvature preserving $\mathfrak{G}_{\mathfrak{p}^{\prime}}$-immersions of $N_{R}$, for $R>1$, for any of these reflective submanifolds.

8.2. Surfaces in $S^{6}$ with $G_{2}$-Frames. Let $i, j, k, x, i x, j x$ and $k x$ be a basis for the imaginary octonions, $\operatorname{Im} \mathbb{O}$, and identify these with $e_{1}, \ldots, e_{7}$, the standard basis for $\mathbb{R}^{7}$. We define the multiplication on the octonions, by the usual quaternionic multiplication on $i, j$ and $k$, and extending the following table by linearity and the relation $a b=-b a$ for all imaginary octonions:

\begin{tabular}{|c|c|c|c|c|c|c|c|}
\hline & $i$ & $j$ & $k$ & $x$ & $i x$ & $j x$ & $k x$ \\
\hline$i x$ & $x$ & $-k x$ & $j x$ & $-i$ & -1 & $-k$ & $j$ \\
\hline$j x$ & $k x$ & $x$ & $-i x$ & $-j$ & $k$ & -1 & $-i$ \\
\hline$k x$ & $-j x$ & $i x$ & $x$ & $-k$ & $-j$ & $i$ & -1 \\
\hline
\end{tabular}

Recall that $G_{2}$ is the Lie group consisting of algebra automorphisms of $\mathbb{O}$. Since an element of $G_{2}$ fixes the identity, it is determined by its (orthogonal, orientation preserving) action on $\operatorname{Im} \mathbb{O}=\mathbb{R}^{7}$; therefore, $G_{2}$ is a subgroup of $S O(7)$. A $G_{2}$-frame is a matrix $F=\left[f_{1}, \ldots, f_{7}\right] \subset G_{2} \subset S O(7)$, whose columns $f_{1}, \ldots, f_{7}$, necessarily satisfy the the same octonionic multiplication table as $e_{1}, \ldots, e_{7}$, described above. That is, $f_{1} f_{2}=f_{3}$, and so on.

There is an almost complex structure $J$ on $S^{6} \subset \mathbb{R}^{7}=\operatorname{Im} \mathbb{O}$, defined by Calabi, [10, as follows: if $u \in S^{6}$, one can identify the tangent space $T_{u} S^{6}$ with the 6-plane in $\mathbb{R}^{7}$ orthogonal to $u$. Then $J_{u}: T_{u} S^{6} \rightarrow T_{u} S^{6}$ is defined by right multiplication by $u$. This is an (orthogonal) linear transformation which satisfies $J_{u}^{2}=-1$, and therefore an almost complex structure. 
8.3. Complex Curves in $S^{6}$. A surface in $S^{6}$ whose tangent space is invariant under the almost complex structure described above is called an (almost) complex curve in $S^{6}$. If $f: M \rightarrow S^{6}$ is an immersed complex curve, one way to choose an adapted $G_{2}$-frame, $F: M \rightarrow G_{2}$ is,

$$
F=\left[f, n_{1}, n_{2}, n_{3}, n_{4}, e_{1}, e_{2}\right],
$$

where $n_{i}$ are normal, and $e_{i}$ are tangent to the surface. For such a matrix in $G_{2}, e_{i} f= \pm e_{j}$.

A study of complex curves in $S^{6}$ can be found in 8]. They have been studied recently as an integrable system associated to primitive maps into a 6-symmetric space $G_{2} / T^{2}$, in [16].

8.4. Special Surfaces from the Three Involution Loop Group. Let us now apply the construction of Section 4.1 to the group $G_{2}$, that is, we wish to interpret elements of $\mathcal{M C}\left(M, \mathcal{H} / \mathcal{H}^{0}\right)_{-1}^{1}$, where $\mathcal{H}=\Lambda\left(G_{2}^{\mathbb{C}}\right)_{\rho \sigma \tau}$, and $\rho, \sigma$ and $\tau$ are defined, respectively, by the extensions (5), of complex conjugation, and the extensions (6) and (7) of

$$
\begin{array}{r}
\sigma=A d_{P}, \quad P=\operatorname{diag}\left(I_{3},-I_{4}\right), \\
\tau=A d_{Q}, \quad Q=\operatorname{diag}\left(1,-I_{2}, I_{2},-I_{2}\right) .
\end{array}
$$

The subspaces $\mathfrak{k}^{\prime}=\mathfrak{k} \cap \mathfrak{u}_{+}$and $\mathfrak{p}^{\prime}=\mathfrak{p} \cap \mathfrak{u}_{-}$consist of the elements of $\mathfrak{g}_{2}$ which have non-zero entries as follows:

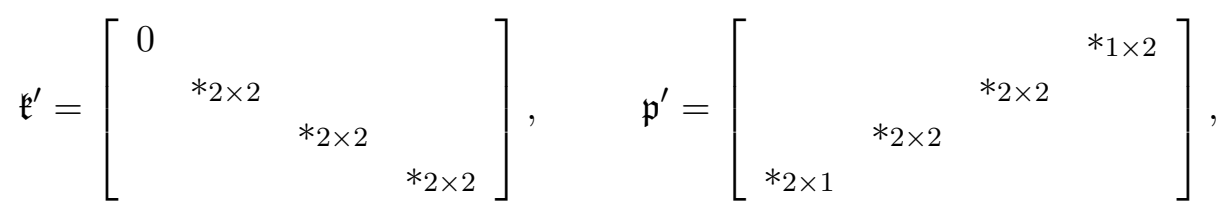

Since right multiplication by an element of $K^{\prime}$ fixes the first column of a matrix $F \in G_{2}$, there is a natural projection of an element $F \in G_{2} / K^{\prime}$ onto $S^{6}=G_{2} / S U(3)$, given by the taking this column. Because $\mathcal{H}^{0}=K^{\prime}$, an element $F \in \mathcal{M C}\left(M, \mathcal{H} / \mathcal{H}^{0}\right)_{-1}^{1}$ can, for real values of $\lambda$, be interpreted, via this projection, as a $G_{2}$-frame for a certain map $f: M \rightarrow S^{6}$. Write the frame as

$$
F=[f, N, X, Y]=\left[f, N_{1}, N_{2}, X_{1}, X_{2}, Y_{1}, Y_{2}\right],
$$

where $f$ is a single column and $N, X$ and $Y$ consist of two columns each. The last 6 columns of $F$ form an orthonormal basis for the total bundle $\left.T S^{6}\right|_{M}$, and the action by $K$ on the right of such a frame preserves the 2-dimensional subspaces spanned by $N, X$ and $Y$ respectively. Thus, given that the frame is normalized to the identity at some point, there is also a natural decomposition of $\left.T S^{6}\right|_{M}$ into these three 2-dimensional sub-bundles,

$$
\left.T S^{6}\right|_{M}=\eta_{1} \oplus \eta_{2} \oplus \eta_{3}
$$

Further, each sub-bundle, $\eta_{i}$, has an almost complex structure on it, inherited from $S^{6}$, because the $\left\{f, N_{1}, N_{2}\right\},\left\{f, X_{1}, X_{2}\right\}$ and $\left\{f, Y_{1}, Y_{2}\right\}$ are all associative triples. 
Now if $F \in \mathcal{M C}(M, \mathcal{H})_{-1}^{1}$, then the Maurer-Cartan form of $F$ is of the form

$$
F^{-1} \mathrm{~d} F=\left[\begin{array}{cccc}
0 & 0 & -\left(\lambda-\lambda^{-1}\right) \theta_{1}^{t} & -\left(\lambda+\lambda^{-1}\right) \theta_{2}^{t} \\
0 & \omega_{1} & \left(\lambda+\lambda^{-1}\right) \beta_{1} & \left(\lambda-\lambda^{-1}\right) \beta_{2} \\
\left(\lambda-\lambda^{-1}\right) \theta_{1} & -\left(\lambda+\lambda^{-1}\right) \beta_{1}^{t} & \omega_{2} & 0 \\
\left(\lambda+\lambda^{-1}\right) \theta_{2} & -\left(\lambda-\lambda^{-1}\right) \beta_{2}^{t} & 0 & \omega_{3}
\end{array}\right],
$$

where $\beta_{i}$ and $\omega_{i}$ are all $2 \times 2$ submatrices and $\theta_{i}$ are $2 \times 1$ column submatrices. Noting that

$$
F^{-1} \mathrm{~d} F=\left[\begin{array}{cccc}
f^{t} \mathrm{~d} f & f^{t} \mathrm{~d} N & f^{t} \mathrm{~d} X & f^{t} \mathrm{~d} Y \\
N^{t} \mathrm{~d} f & N^{t} \mathrm{~d} N & N^{t} \mathrm{~d} X & N^{t} \mathrm{~d} Y \\
X^{t} \mathrm{~d} f & X^{t} \mathrm{~d} N & X^{t} \mathrm{~d} X & X^{t} \mathrm{~d} Y \\
Y^{t} \mathrm{~d} f & Y^{t} \mathrm{~d} N & Y^{t} \mathrm{~d} X & Y^{t} \mathrm{~d} Y
\end{array}\right]
$$

it follows from (30) that the bundle $\eta_{1}$ is a sub-bundle of the normal bundle, that $\omega_{1}, \omega_{2}$ and $\omega_{3}$ are the connections for the bundles $\eta_{1}, \eta_{2}$ and $\eta_{3}$ respectively, and that $\left[\left(\lambda-\lambda^{-1}\right) \theta_{1},\left(\lambda+\lambda^{-1}\right) \theta_{2}\right]^{t}$ is essentially the coframe.

Note that $\mathfrak{u}_{-}$consists of all matrices in $\mathfrak{g}_{2}$ of the form

$$
\left[\begin{array}{cc}
0 & *_{3 \times 4} \\
* 3 \times 4 & 0
\end{array}\right]
$$

so it follows from Lemma 5.1 that solutions constructed from 2-dimensional regular curved flats via the KDPW method will generically project to regular maps into $S^{6}$.

At $\lambda=1$ we have

$$
\left.F^{-1} \mathrm{~d} F\right|_{\lambda=1}=\left[\begin{array}{cccc}
0 & 0 & 0 & -2 \theta_{2}^{t} \\
0 & \omega_{1} & 2 \beta_{1} & 0 \\
0 & -2 \beta_{1}^{t} & \omega_{2} & 0 \\
2 \theta_{2} & 0 & 0 & \omega_{3}
\end{array}\right]
$$

Comparing with (31), this implies that, for this value of $\lambda, X_{1}$ and $X_{2}$ also lie in the normal bundle, so the tangent space is spanned by $Y_{1}$ and $Y_{2}$. Hence, $\left.f\right|_{\lambda=1}$ is a complex curve. Moreover, the second fundamental form for $\left.f\right|_{\lambda=1}$ is given by the matrix $\left[N^{t} \mathrm{~d} Y, X^{t} \mathrm{~d} Y\right]^{t}$ and this is zero. Thus $\left.f\right|_{\lambda=1}$ is a totally geodesic complex curve in $S^{6}$.

We now examine the conditions we get for $f_{\lambda}$ from the equation (9). It is easy to verify that, in this case, the condition is equivalent to the three equations

$$
\begin{array}{r}
\mathrm{d} \omega_{1}+\omega_{1} \wedge \omega_{1}=4 \beta_{1} \wedge \beta_{1}^{t}, \\
\mathrm{~d} \omega_{2}+\omega_{2} \wedge \omega_{2}=4 \beta_{1}^{t} \wedge \beta_{1}, \\
\mathrm{~d} \omega_{3}+\omega_{3} \wedge \omega_{3}=4 \theta_{2} \wedge \theta_{2}^{t} .
\end{array}
$$

These equations say that the bundle-connection pairs $\left(\eta_{i}, \nabla_{i}\right)$, where the connection is induced from $S^{6}$, are isomorphic to those obtained at $\lambda=1$. 


\section{FUNDING}

Research supported by the Japan Society for the Promotion of Science.

\section{ACKNOWLEDGEMENTS}

Thanks are due to Josef Dorfmeister, Daniel Fox, Jost-Hinrich Eschenburg and Martin Guest, for helpful advice at various critical moments during the preparation of this manuscript.

\section{REFERENCES}

[1] M. J. Ablowitz, D. J. Kaup, A. C. Newell, and H. Segur. Method for solving the sine-Gordon equation. Phys. Rev. Lett., 30:1262-1264, 1973.

[2] M. Adler and P. van Moerbeke. Completely integrable systems, Euclidean Lie algebras, and curves. Adv. in Math., 38:267-317, 1980.

[3] M. Adler and P. van Moerbeke. Linearization of Hamiltonian systems, Jacobi varieties and representation theory. Adv. in Math., 38:318-379, 1980.

[4] J. Berndt, J. H. Eschenburg, H. Naitoh, and K. Tsukada. Symmetric submanifolds associated with irreducible symmetric R-spaces. Math. Ann., 332:721-737, 2005.

[5] D. Brander. Curved flats, pluriharmonic maps and constant curvature immersions into pseudo-Riemannian space forms. arxiv preprint:0610058. To appear in: Ann. Global Anal. Geom.

[6] D. Brander and J. Dorfmeister. The generalized DPW method and an application to isometric immersions of space forms. arxiv preprint:060424\%, 2006.

[7] M. Brück, X. Du, J. Park, and C. L. Terng. The submanifold geometries associated to grassmannian systems. Mem. Amer. Math. Soc. 155, (735):viii + 95 pp., 2002.

[8] R. L. Bryant. Submanifolds and special structures on the octonians. J. Differential Geom., 17(2):185-232, 1982.

[9] F. E. Burstall and F. Pedit. Harmonic maps via Adler-Kostant-Symes theory. In Harmonic maps and integrable systems, number E23 in Aspects of Mathematics. Vieweg, 1994.

[10] E. Calabi. Construction and properties of some 6-dimensional almost complex manifolds. Trans. Amer. Math. Soc., 87:407-438, 1958.

[11] J. Dorfmeister, F. Pedit, and H. Wu. Weierstrass type representation of harmonic maps into symmetric spaces. Comm. Anal. Geom., 6:633-668, 1998.

[12] D. Ferus and F. Pedit. Curved flats in symmetric spaces. Manuscripta Math., 91:445454, 1996.

[13] D. Ferus and F. Pedit. Isometric immersions of space forms and soliton theory. Math. Ann., 305:329-342, 1996.

[14] R. Harvey and H. B. Lawson. Calibrated geometries. Acta Math., 148:47-157, 1982.

[15] D. Hilbert. Ueber Flachen von constanter Gausscher Krummung. Trans. Amer. Math. Soc., 2:87-99, 1901.

[16] S. Kong, C. L. Terng, and E. Wang. Associative cones and integrable systems. Chinese Ann. Math. Ser. B, 27(2):153-168, 2006.

[17] B. Kostant. The solution to a generalized Toda lattice and representation theory. Adv. in Math., 34:195-338, 1979.

[18] I. M. Krichever. An analogue of the d'Alembert formula for the equations of a principal chiral field and the sine-Gordon equation. Dokl. Akad. Nauk SSSR, 253(2):288292, 1980.

[19] D. S. P. Leung. On the classification of reflective submanifolds of Riemannian symmetric spaces. Indian Univ. Math. J., 24:327-339, 1974. 
[20] D. S. P. Leung. Errata: "On the classification of reflective submanifolds of Riemannian symmetric spaces". Indian Univ. Math. J., 24:1199, 1975.

[21] D. S. P. Leung. Reflective submanifolds. III. congruency of isometric reflective submanifolds and corrigenda to the classification of reflective submanifolds. J. Differential Geom., 14, 1979.

[22] J. D. Moore. Isometric immersions of space forms in space forms. Pacific J. Math., 40:157-166, 1972.

[23] H. Naitoh. Symmetric submanifolds of compact symmetric spaces. Tsukuba J. Math., 10:215-242, 1986.

[24] H. Naitoh. Compact simple lie algebras with two involutions and submanifolds of compact symmetric spaces I, II. Osaka J. Math., 30, 1993.

[25] H. Naitoh. Grassman geometries on compact symmetric spaces of general type. $J$. Math. Soc. Japan, 50, 1998.

[26] H. Naitoh. Grassman geometries on compact symmetric spaces of classical type. Japanese J. Math., 26, 2000.

[27] H. Naitoh. Grassman geometries on compact symmetric spaces of exceptional type. Japanese J. Math., 26, 2000.

[28] A. Pressley and G. Segal. Loop Groups. Oxford Math. monographs. Clarendon Press, Oxford, 1986.

[29] H. Reckziegel. Horizontal lifts of isometric immersions into the bundle space of a pseudo-Riemannian submersion. Global differential geometry and global analysis (1984), Lecture Notes Math., 1156:264-279, 1985.

[30] W. W. Symes. Systems of Toda type, inverse spectral problems, and representation theory. Invent. Math., 59:13-51, 1980.

[31] K. Tenenblat and C. L. Terng. Baecklund's theorem for $n$-dimensional submanifolds of $\mathbb{R}^{2 n-1}$. Ann. Math., 111:477-490, 1980.

[32] C. L. Terng. A higher dimensional generalisation of the sine-Gordon equation and its soliton theory. Ann. Math., 111:491-510, 1980.

[33] C. L. Terng. Geometries and symmetries of soliton equations and integrable elliptic equations. arXiv preprint:0212372, 2002.

Department of Mathematics,, Faculty of Science, Kobe University,, 1-1 ROKKODAI, NADA-KU,, KoBE 657-8501, JAPAN

E-mail address: brander@math.kobe-u.ac.jp 